\title{
Modeling and Analysis of Data Harvesting Architecture based on Unmanned Aerial Vehicles
}

\author{
Chang-Sik Choi, François Baccelli, and Gustavo de Veciana
}

\begin{abstract}
This paper explores an emerging wireless Internetof-things (IoT) architecture based on unmanned aerial vehicles (UAVs). We consider a network where a fleet of UAVs at a fixed altitude flies on planned trajectories and IoT devices on the ground are scheduled to transmit their data to the UAVs when the latter are nearby. In such a system, the UAVs' motion triggers the uplink transmissions of the IoT devices. As a result, network performance is determined by the geometric and dynamic characteristics of the system. We propose a joint stationary model for UAVs and IoT devices and then evaluate the interference, the coverage probability, and the data rate of the typical UAV. To assess the harvesting capability of the proposed architecture, we derive a formula for the amount of data uploaded from each IoT device to a UAV. We also establish a linear relationship between the UAV coverage and the harvesting capability of the network, which provides insights into the design of the proposed harvesting scheme. In addition, we use our analytical results to numerically show that there exists a trade-off between the uploaded data and the size of the IoT scheduling window. Specifically, for a given UAV and IoT geometry, there exists an optimal scheduling window that maximizes the harvesting capability of the proposed network.
\end{abstract}

\section{INTRODUCTION}

\section{A. Motivation and Related Work}

This paper investigates a wireless data harvesting architecture for static ground-based Internet-of-Things devices (IoT) [2]-[4]. IoT devices may have various functions such as performing environmental measurements from some area, measuring electric and water systems, collecting average road traffic counts, and monitoring extreme environments [5][7]. The data continuously generated from these IoT devices are uploaded to the Internet using technologies such as narrowband-IoT [8], [9] or low-power-wide-area networks [10]-[12]. Currently, these approaches rely on the existence of fixed network infrastructure, e.g., base stations or access points. Consequently, the performance of such technologies is often limited by the bottlenecks around infrastructure nodes [13], [14]. When the density of IoT devices varies across areas, deploying more base stations may be required for improving performance. Installing denser infrastructure might be impractical due to technological and financial reasons.

One possible alternative to collect IoT data without increasing the fixed network infrastructure is to leverage mobile

Chang-sik Choi is a senior system engineer of Qualcomm Inc, Bridgewater, New Jersey. Francois Baccelli is with Department of Electrical and Computer Engineering and Department of Mathematics, The University of Texas at Austin, TX, USA. Gustavo de Veciana is with Department of Electrical and Computer Engineering, The University of Texas at Austin, TX, USA. (emails: chang-sik.choi@utexas.edu, baccelli@math.utexas.edu, gustavo@ece.utexas.edu)

A short version is in Proc. IEEE ISIT 2019 [1] agents, e.g., vehicles [14]-[20] which visit IoT devices and collect their data. When the vehicles arrive at stations connected to the network, the data will be uploaded to the Internet. This vehicle harvesting concept requires a delay-tolerant setting, where some delay is accepted [21]-[27] in order to improve network capacity or reduce aggregate interference. As discussed in [2]-[4], a number of IoT applications tolerate such delays. For such applications, relying on mobile vehicle harvesters is a viable and economical option to collect IoT data.

Nevertheless, IoT data harvesting based on mobile vehicles suffers of a few practical restrictions. For instance, vehicles travel only on the existing roads and thus some IoT devicessuch as those with short transmission range or located in rural areas with sparse road networks - may not be able to establish reliable connections to nearby vehicles at any time [28], [29]. As an alternative to vehicle harvesters, UAVs are considered as a way to extend mobile data harvesting beyond the existing road systems [30]-[32]. In practice, certain regulations restrict the use of UAVs. For instance, UAVs operated for recreational purposes are not allowed to enter no-flying zones or to operate near groups of people, public events, or a stadium. In addition, in the United States, pilots of UAV operated for commercial usage must be certified by the Federal Aviation Administration [33]. Even when these restrictions apply, UAV configured to meet the needs of telecommunication operators could become an effective way to achieve large-scale connectivity with a marginal infrastructure support, particularly so in areas with a sparse road system and limited wired backhaul networks. Here are a few concrete examples of such UAV networks.

- A fleet of UAVs monitoring a large area where small sensor devices are constantly collecting seismic activity data [5];

- A platoon of military UAVs flying over a wide area to collect scattered information from IoT sensors on the battle ground [34];

- Intelligent transportation system UAVs flying on certain streets and harvesting traffic or sensor data from infrastructure such as roadside units [7], [35].

In these examples and many others, the network elements consist of UAVs and static data devices. These data devices are deployed over a large area where fixed infrastructure is unavailable or undesirable. These sensors or devices persistently produce data and a UAV fleet is operated to collect them.

The literature on wireless networks relying on UAVs is quite rich, with contributions stemming both from industry stakeholders and academic researchers [30]-[32], [34]-[41]. 
Stochastic geometry was used to study the performance of UAV-assisted cellular networks and device-to-device networks in [42]-[45]. UAV downlink/uplink performance was also analyzed in [36], [45].

Compared to this literature, the first novelty of the present paper is the new parametric stochastic geometry model allowing one to represent architectures based on fleets of operated UAVs. This is probably the simplest, though not simplistic, parametric model in this class as UAV motion is linear and with constant velocity rather than adaptive and linked to traffic. The second novelty is the analytic framework based on the evaluation of the interference field statistics which allows us to derive expressions for various classical metrics, such as the probability of coverage, and also for less classical ones such as the mean amount of data harvested from each IoT device as UAVs progress. To the best of our knowledge, the last metric was never been analyzed. The third novelty is the optimization of the harvesting capability as a function of the network parameters.

\section{B. Contributions}

A parametric analytical framework for UAV harvesting networks: We propose a parametric model for data harvesting architectures where UAVs collect delay-tolerant data from static surface-level IoT devices. UAVs are designed to cover a wide area, with no need of fixed infrastructure, e.g., base stations. At any time, every UAV receives data transmission from one of the IoT devices located in a rectangle activation window of size $w$ by $l$ on the ground. The fleet of UAVs is assumed to move at constant speed $v$ in a coordinated way. As the fleet of UAVs progresses, the windows of UAVs also progress on the ground. The parameters as set to values such that the proposed architecture provides universal coverage of IoT devices, that is, each device is periodically covered by some UAV (rather than always covered as in the fixed infrastructure architecture case). Parametric stationary point processes are used to model the locations of IoT devices and UAVs, respectively. Specifically, the locations of the UAVs are modeled by a stationary grid characterized by inter-point distances $\mu$ between UAVs on any given line and some common altitude $h$. The locations of IoT devices on the ground are modeled by an independent planar Poisson point process with a fixed intensity.

Performance analysis: We first use Palm calculus to derive the Laplace transform of the interference seen by a typical UAV. We show that interference distribution is time-invariant. We also derive the distribution of the signal-to-interference (SIR) ratio of the typical UAV and the distribution of its data rate. Similarly, we obtain an expression for the mean value of the amount of harvested data transmitted from the typical IoT device to a UAV when the latter passes over the IoT device location. We use the mass transport principle to link the mean value of the harvested data and the instantaneous UAV data rate.

Network performance trade-off As explained above the fact that UAVs are in motion, allows such an architecture to provide universal (though intermittent) coverage with a

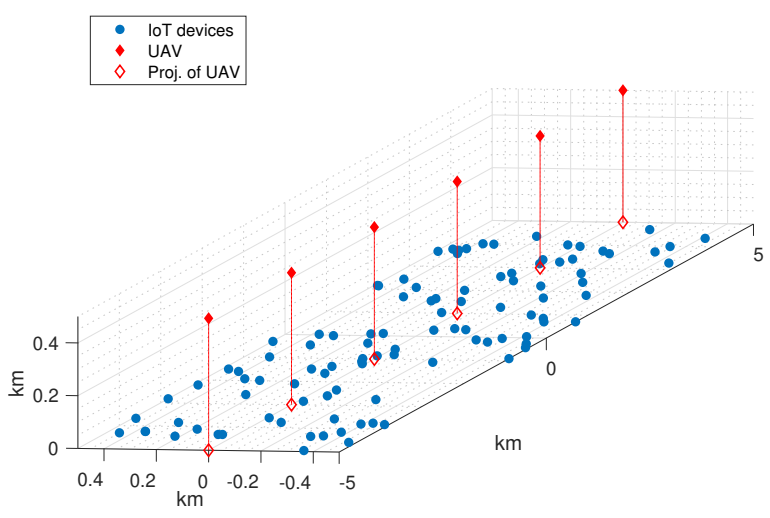

Fig. 1. Illustration of the proposed IoT harvesting network where the UAVs are located at the altitude $z=0.5 \mathrm{~km}$ and the distance between UAVs is given by $2 \mathrm{~km}$. The platoon of UAVs is assumed to move in the positive $x$ axis with speed $v$. The activation window of each UAV is characterized by a rectangle $w \times l$ centered at the projection of the UAV on the plane. See Fig. 2 for the activation window.

smaller number of UAVs than what would be needed in a static architecture based on infrastructure nodes. (specifically, in the system model, the window size $w$ is set to be less than $\mu$ ). Furthermore, thanks to inherent mobility, UAVs are close to the IoT devices when transmissions occur, increasing the received signal power. Similarly, UAVs can be operated at a safe distance from each other, reducing the interference. In this sense, the window size $w$ is one of the key parameters that characterize the performance of the proposed motionbased harvesting architecture. We show that there is a trade-off relationship between the data rate of the typical UAV and the size of the window. More precisely, for all given densities of IoT devices, there exists a unique value $w^{\star}$ that maximizes both the data rate of the typical UAV and the harvesting capability of the network.

\section{SySTEM MODEL}

This section introduces the system model of the proposed network architecture. Then, access control, propagation model, and network performance metrics are discussed. In order to ease the exposition, we start with the linear case (a single line of UAVs progressing a speed $v$ along this line, and a strip of IoT devices along this line) and postpone the description of the planar case (an infinite number of parallel lines covering the plane, each with UAVs as above, with a homogeneous planar point process of IoT devices) to the end of the paper.

\section{A. Moving UAV Fleet and Stationary IoT Devices}

As mentioned in the introduction, this paper focuses on the data-harvesting applications of a UAV fleet with a idealized motion planned by some operator. These UAVs are separated by some distance while in the air to avoid collision. Thus, we use periodic points on a line parallel to the $x$-axis in order to model the UAVs in the air. The distance between the points 
is given by $\mu$ and the altitude of the UAVs is given by $h$. To address the randomness of the UAVs at time 0 , the periodic points are shifted by a single uniform vector, $(U, 0,0)$ where $U \sim$ Uniform $\left[-\frac{\mu}{2}, \frac{\mu}{2}\right]$.

$$
\Psi(0)=\sum_{k \in \mathbb{Z}} \delta_{(k \mu, 0, h)+(U, 0,0)},
$$

where $\delta_{x}$ denotes the Dirac measure indicating a point mass at location $x$, and $\mathbb{Z}$ denotes the set of all integers. This model is probably the simplest model for the planned movements of a UAV fleet. For analytical tractability, we assume that all UAVs move at the same speed $v$ in the positive $x$-axis. Then, the locations of UAVs at time $t>0$ are given by

$$
\Psi(t)=\sum_{k \in \mathbb{Z}} \delta_{(k \mu, 0, h)+(U, 0,0)+(v t, 0,0)} .
$$

IoT devices are assumed to be static and distributed according to an independent planar Poisson point process with intensity $\lambda$ on a strip. The finite transmission range of IoT devices is captured by having the Poisson IoT devices restricted to a strip of width $l$ centered at the $x$-axis. The proposed model allows us to specify the properties of uplink transmissions from IoT devices to UAVs as the latter progress. Fig. 11 illustrates the UAVs, their projections onto the plane, and the IoT devices. Thanks to the random shift $U$, the proposed UAV point process is stationary (w.r.t. shifts along the $x$-axis) and even jointly stationary with the device point process [46].

Remark 1: When the UAVs are assumed to move in the positive direction of the $x$-axis with speed $v$, the UAV point process at time $t>0$ is the initial UAV point process at time 0 , shifted by a vector $(v t, 0,0)$. The proposed UAV point process is translation-invariant w.r.t. motion along the $x$-axis, i.e., the distributions of the UAV point process and of its shifted version are the same: $\Phi(t) \stackrel{d}{=} \Phi(0)$. The translationinvariant structure provides the mathematical justification for the definition of the typical UAV thanks to Palm calculus. The statistics of coverage and rate of the typical UAV will be evaluated in Section III

\section{B. Uplink Transmissions Associated With Motion}

In the proposed network, uplink transmissions from IoT devices are triggered by the motion of UAVs. We assume that an IoT device is marked as active if and only if it is located inside the coverage area of an UAV, which will be referred to as its activation windows. The activation window is modeled by the set $\left[-\frac{w}{2}, \frac{w}{2}\right] \times\left[-\frac{l}{2}, \frac{l}{2}\right]$ on the plane, centered at the projection of every UAV onto the plane.

The geometry of the activation window determines key feature of the proposed architecture, and in particular the period of the activation process and the properties of the associated uplink transmissions. As the UAV fleet moves in the positive $x$-direction, the activation windows also move. As a result, UAVs are able to harvest the data from all IoT

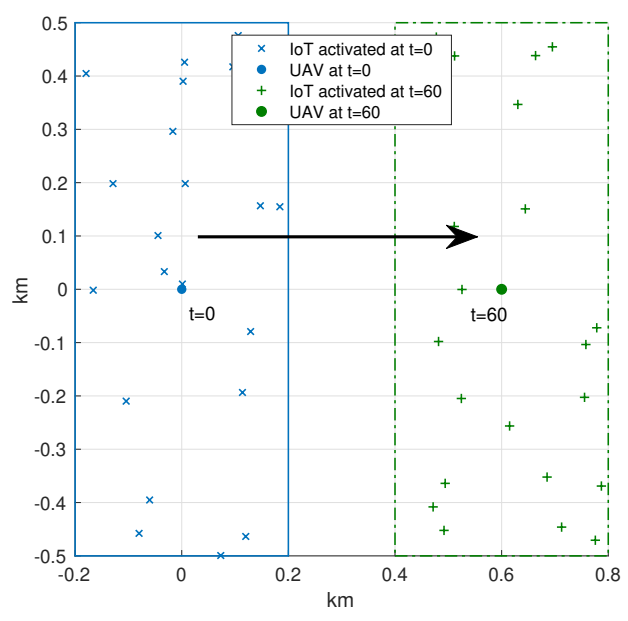

Fig. 2. Illustration of the proposed network with a UAV located at $(0,0, h)$ at time zero. The black arrow indicates the direction of UAV motion. Each UAV moves at $36 \mathrm{~km} / \mathrm{h}$. The density of IoT devices is $100 / \mathrm{km}^{2}$. The activation windows at time $t=0,60$ are indicated by the solid blue and dotted green rectangles, respectively. At each time slot, only one IoT device can upload its data to the corresponding UAV. The access-granted IoT device at a certain time slot is uniformly selected in every window.

devices on the strip. This is what we called universal service above. The windows at time $t$ are

$$
\begin{aligned}
\mathcal{W}(t) & =\bigcup_{i \in \mathbb{Z}} \mathcal{W}_{i}(t) \\
& =\bigcup_{i \in \mathbb{Z}}\left[\mu i+v t-\frac{w}{2}, \mu i+v t+\frac{w}{2}\right] \times\left[-\frac{l}{2}, \frac{l}{2}\right] .
\end{aligned}
$$

To analyze the network performance, time is assumed to be slotted. We assume that at each time slot, a single IoT device is randomly chosen from each window for the uplink transmission (we recall that windows do not intersect at any given time). Hence, at each time, all IoT devices inside the window of an UAV are activated as possible candidates for uplink transmission, and then at most one IoT device is granted access for uplink transmission. Such an access control model can be modeled by time division multiple access (TDMA) scheduling per UAV window, which will later be approximated by a processor sharing scheme, which is equivalent to assuming that the time slot is very small compared to the time an IoT device spends in the window of a passing by UAV. The TDMA scheduling is assumed to cope with the fact that the transmit power of uplink transmitters is limited and the network performance is interference limited. Fig. 2 illustrates the locations of IoT devices, associated activation windows at time 0 and 20, and the corresponding active IoT devices, respectively.

\section{Propagation Model}

Time is slotted and each time slot has a duration $T_{s}$. Each time slot is assumed to be equal to the coherence time $T_{c}$ of the wireless channel. In other words, we assume that each slot experiences a different realization of a random fading.

To analyze the network performance, the received signal power is modeled by a distance-based power law path loss 
TABLE I

Network Model ANd Parameters

\begin{tabular}{|c|c|}
\hline Network parameters & Description \\
\hline IoT devices & $\Phi \sim$ Planar Poisson $\lambda$ on the strip \\
\hline UAVs & Shifted comb process of inter distance $\mu$ \\
\hline UAV altitude & $h$ \\
\hline UAV activation window & $w \times l$ on the plane \\
\hline Multiple access & Time-division multiple access \\
\hline UAV motion and speed & $x$-axis with speed $v$ \\
\hline Path loss exponent & $\alpha>1$ \\
\hline UAV-IoT fading & Gamma $(m, \Omega / m)$ \\
\hline
\end{tabular}

model with Nakagami-m fading with parameters $m$ and $\Omega$. To maintain the analytical tractability of the paper, we do not consider air to ground channel model with direct line of sight componen ${ }^{1}$. The Nakagami-m fading is generally used to model various fading conditions including Rayleigh. For instance, if $m=\Omega=1$, Nakagami-m fading boils down to Rayleigh. The received signal power at distance $d$ is given by $p G d^{-\alpha}$, where $p$ is the transmit power, $G$ is a Gamma random variable, $G \sim \operatorname{Gamma}(m, \Omega / m)$, and $\alpha>1$ is the path loss exponent [47].

\section{Performance Metrics}

For the proposed UAV network, we evaluate the performance from two typical perspectives: the UAV's perspective and the IoT device's perspectives. Under the Palm distribution of the UAV point process, we derive the coverage probability: $\mathbf{P}(\operatorname{SIR}>\tau)$ and the distribution of the corresponding instantaneous data rate. Under the Palm distribution of the IoT point process, we derive the amount of data uploaded from a typical IoT device to a UAV.

\section{Performance From the UAVs' Perspective}

\section{A. Shot-noise Seen by a Typical UAV}

The shot-noise process $N(t)$ is a stochastic process whose value at time $t$ is the sum of the signal power received by the typical UAV from all the access-granted IoT devices at time $t$. Due to the randomness of the locations of nodes and that of the wireless channel, $N(t)$ is a random variable that will be characterized by its Laplace transform, $\mathbf{E}[\exp (-s N(t))]$ with $s$ the Laplace argument. The Laplace transform of the shot-noise process has been extensively studied to obtain the key performance metrics such as the coverage probability. In particular, the shot-noise process of the Poisson point process has been used extensively [46]. In this paper, the underlying IoT devices are modeled as a realization of the Poisson point process. However, due to the TDMA processing at each UAV window and the movement of UAVs, the access-granted IoT devices (which determine the shot-noise process) are not distributed according to a Poisson point process. We hence need a specific analysis that is described below.

Consider the Palm distribution of the UAV point process. Without loss of generality, under this Palm distribution, the

\footnotetext{
${ }^{1}$ The performance with line of sight channel component can be derived, leveraging the analysis presented in this paper.
}

typical UAV is located at $(0,0, h)$ at a given time slot [48]. Consequently, the activation windows for all UAVs are

$$
\mathcal{W}=\bigcup_{i \in \mathbb{Z}} \mathcal{W}_{i}=\bigcup_{i \in \mathbb{Z}}\left[\mu i-\frac{w}{2}, \mu i+\frac{w}{2}\right] \times\left[-\frac{l}{2}, \frac{l}{2}\right] .
$$

Under the TDMA scheduling, the shot-noise process seen at the typical UAV is given by

$$
N=\sum_{\left(X_{i}, Y_{i}\right) \in \hat{\Phi}} p G_{i}\left\|\left(X_{i}, Y_{i}, 0\right)-(0,0, h)\right\|^{-\alpha} \mathbb{1}_{\left\{\Phi\left(\mathcal{W}_{i}\right) \neq \emptyset\right\}}
$$

where $\left(X_{i}, Y_{i}\right)$ are the $x$ and $y$ coordinates of the transmitting IoT device, if any, in window $\mathcal{W}_{i}$. The point process $\hat{\Phi}$ denotes the access-granted IoT point process. Let $\mathbb{1}_{\{A\}}$ denote the indicator function that takes value one if $A$ is true, or zero otherwise.

Theorem 1: $\mathcal{L}_{N}(s)$ the Laplace transform of the uplink shotnoise process at the typical UAV is given by Eq. (5) where $s$ is the Laplace transform argument.

Proof: We can write $\mathcal{L}_{N}(s)$ as follows:

$$
\begin{aligned}
& \mathbf{E}_{\Psi}^{0}\left[e^{-s \sum_{\left(X_{i}, Y_{i}\right) \in \mathcal{W}_{i}}^{i \neq 0} p G_{i}\left\|\left(X_{i}, Y_{i}, 0\right)-(0,0, h)\right\|^{-\alpha}} \mathbb{1}_{\left\{\Phi\left(\mathcal{W}_{i}\right) \neq \emptyset\right\}}\right] \\
& \stackrel{(\mathrm{a})}{=} \mathbf{E}_{\Psi}^{0}\left[\prod_{X_{i}, Y_{i}} \mathbf{E}\left[e^{\left.-s p G_{i}\left\|\left(X_{i}, Y_{i},-h\right)\right\|^{-\alpha} \mathbb{1}_{\left\{\phi\left(\mathcal{W}_{i}\right) \neq \emptyset\right\}}\right]}\right],\right.
\end{aligned}
$$

To derive (a), we use the fact that the points from disjoint windows are independent. Using that the number of points in each window follows the Poisson distribution, we have

$$
\begin{aligned}
& \mathcal{L}_{N}(s) \\
& =\mathbf{E}_{\Psi}^{0}\left[\prod _ { i \in \mathbb { Z } } \left(\mathbf{P}\left(\Phi\left(\mathcal{W}_{i}\right)=\emptyset\right)\right.\right. \\
& \left.\left.+\mathbf{P}\left(\Phi\left(\mathcal{W}_{i}\right) \neq \emptyset\right) \cdot \mathbf{E}\left[e^{-s p G_{i}\left\|\left(X_{i}, Y_{i},-h\right)\right\|^{-\alpha}}\right]\right)\right] \\
& \stackrel{(\text { b) }}{=} \mathbf{E}_{\Psi}^{0}\left[\prod _ { i \in \mathbb { Z } } \left(e^{-\lambda w l}\right.\right. \\
& \left.\left.+\left(1-e^{-\lambda w l}\right) \int_{\operatorname{supp}(G)} e^{-s p g\left(X_{i}^{2}+Y_{i}^{2}+h^{2}\right)^{-\frac{\alpha}{2}}} f_{G}(g) \mathrm{d} g\right)\right] \\
& \stackrel{(\mathrm{c})}{=} \prod_{i \in \mathbb{Z}}\left(e^{-\lambda w l}+\frac{1-e^{-\lambda w l}}{w l}\right. \text {. } \\
& \left.\int_{i \mu-\frac{w}{2}}^{i \mu+\frac{w}{2}} \int_{-\frac{l}{2}}^{\frac{l}{2}} \int_{\operatorname{supp}(G)} e^{-\operatorname{spg}\left(x^{2}+y^{2}+h^{2}\right)^{-\frac{\alpha}{2}}} f_{G}(g) \mathrm{d} g \mathrm{~d} y \mathrm{~d} x\right) \\
& \stackrel{(\mathrm{d})}{=} \prod_{i \in \mathbb{Z}}\left(e^{-\lambda w l}+\frac{1-e^{-\lambda w l}}{w l}\right. \text {. } \\
& \left.\int_{i \mu-\frac{w}{2}}^{i \mu+\frac{w}{2}} \int_{-\frac{l}{2}}^{\frac{l}{2}}\left(\frac{1}{1+\frac{s p \Omega m^{-1}}{\left(x^{2}+y^{2}+h^{2}\right)^{\frac{\alpha}{2}}}}\right)^{m} \mathrm{~d} y \mathrm{~d} x\right) .
\end{aligned}
$$

To derive (b), we use the fact that the probability that the window $\mathcal{W}_{i}$ is empty of points is equal to $\exp (-\lambda w l)$. To derive (c), we use the fact that, given that the window is not empty of points, a randomly selected point is uniformly distributed inside the window $\mathcal{W}_{i}=\left[i \mu-\frac{w}{2}, i \mu+\frac{w}{2}\right] \times\left[-\frac{l}{2}, \frac{l}{2}\right]$, 


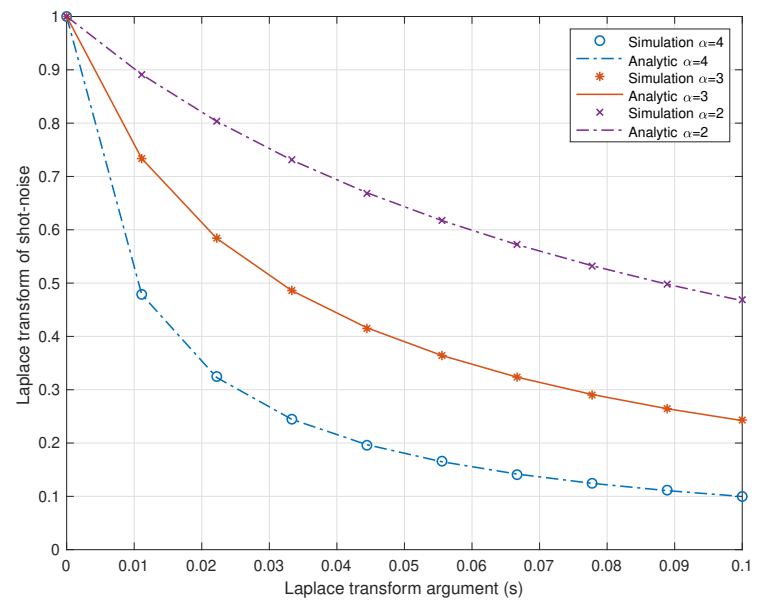

Fig. 3. Laplace transform of the shot-noise process seen by the typical UAV with Nakagami-m fading parameters $m=\Omega=1$. We consider $\lambda=1000 / \mathrm{km}^{2}, \mu=2 \mathrm{~km}, w=0.25 \mathrm{~km}, l=0.5 \mathrm{~km}$, and $h=0.25$ $\mathrm{km}$. Simulation results exactly match the analytically derived formula.

and the selected points across windows are also independent. To get (d), we use the Laplace transform of the exponential random variable with mean one.

Note that the integral formula, Eq. (5), is not equal to zero because (1) the shot-noise process $N$ is statistically dominated by the shot-noise process seen from the origin and (2) the Laplace transform of the planar Poisson shot-noise process is not equal to zero. See [46], [49] for the Laplace transform of the planar Poisson shot-noise process.

Fig. 3 illustrates the shot-noise process obtained from the derived formula and from Monte Carlo simulations, respectively. It confirms that the derived formula is accurate. The figure shows that the Laplace transform decreases as the path loss exponent increases.

Remark 2: The shot-noise process of the typical UAV is time-invariant. Note that the time-invariance property of the shot-noise process comes from the fact that the underlying IoT point process is modeled by a planar Poisson point process and the fact that the UAV point process is translation and timeinvariant. The shot-noise process determines the interference seen by the typical UAV (defined as the sum of the powers of all signals received by the typical UAV except that of the IoT device that transmits to it at that time), which in turn determines the SIR or SINR and the link performance.

Corollary 1: $\mathcal{L}_{I}(s)$ the Laplace transform of the interference seen by the typical UAV is given by Eq. 6.

The activation window $[-w / 2, w / 2] \times[-l / 2, l / 2]$ is used to schedule the transmissions of IoT devices. For the evaluation of interference, the signal component from the window $\mathcal{W}_{0}$ is removed from Eq. (1).

Example 1: For the parameters $m=\Omega=1$, Nakagami fading boils down to Rayleigh. In this case, $\mathcal{L}_{\bar{I}}(s)$ the Laplace transform of the shot-noise is given by Eq. (7)

\section{B. Coverage Probability of the Typical UAV}

We assume that each UAV decodes the received signal transmitted from its own window, by treating interference from other windows as noise. The coverage probability is defined as a function of $\tau: \mathbf{P}_{\Psi}^{0}(\mathrm{SIR} \geq \tau)$ under the Palm distribution of $\Psi$, where $\mathrm{SIR}=S /(I+n)$ and the random variables $S$ and $I$ denote the received signal power and the received interference power, respectively.

Theorem 2: The coverage probability of the typical UAV is

$$
\frac{1-e^{-\lambda w l}}{w l} \int_{-\frac{w}{2}}^{\frac{w}{2}} \int_{-\frac{l}{2}}^{\frac{l}{2}} \underbrace{\sum_{i=0}^{m-1} \frac{(-s)^{i}}{i !} \frac{\mathrm{d}^{i}}{\mathrm{~d} s^{i}} \mathcal{L}_{I}(s)}_{\text {(a) }} \mathrm{d} y \mathrm{~d} x
$$

where term (a) being evaluated at $s=\frac{\tau m\left(x^{2}+y^{2}+h^{2}\right)^{\frac{\alpha}{2}}}{p \Omega}$ and $\mathcal{L}_{I}(s)$ is the Laplace transform of the interference at the typical UAV provided in Eq. (6).

Proof: First, the coverage probability of the typical UAV is derived under the Palm distribution of the UAV point process, as in the derivation of the shot-noise process. The coverage probability of the typical UAV is given by

$$
\begin{aligned}
& \mathbf{P}_{\Psi}^{0}(\operatorname{SIR} \geq \tau) \\
& =\mathbf{P}_{\Psi}^{0}\left(\operatorname{SIR} \geq \tau \mid \Phi\left(\mathcal{W}_{0}\right) \neq \emptyset\right) \mathbf{P}\left(\Phi\left(\mathcal{W}_{0}\right) \neq \emptyset\right) \\
& \quad+\underbrace{\mathbf{P}_{\Psi}^{0}\left(\operatorname{SIR} \geq \tau \mid \Phi\left(\mathcal{W}_{0}\right)=\emptyset\right)}_{(\text {a) }} \times \mathbf{P}_{\Psi}^{0}\left(\Phi\left(\mathcal{W}_{0}\right)=\emptyset\right) \\
& =\mathbf{P}_{\Psi}^{0}\left(\operatorname{SIR} \geq \tau \mid \Phi\left(\mathcal{W}_{0}\right) \neq \emptyset\right) \mathbf{P}\left(\Phi\left(\mathcal{W}_{0}\right) \neq \emptyset\right),
\end{aligned}
$$

where we use the fact that the coverage probability is considered to be zero if the window $\mathcal{W}_{0}$ is empty of points; term (a) vanishes. Let $X_{0}$ and $Y_{0}$ denote the $x, y$ coordinates of the transmitting IoT device in the window of the typical UAV, $\mathcal{W}_{0}$, respectively. Then, we have

$$
\begin{aligned}
& \mathbf{P}\left(\operatorname{SIR} \geq \tau \mid \Phi\left(\mathcal{W}_{0}\right) \neq \emptyset\right) \\
& =\mathbf{P}_{\Psi}^{0}\left(\frac{p G\left\|\left(X_{0}, Y_{0},-h\right)\right\|^{-\alpha}}{\sum_{\left(X_{i}, Y_{i}\right) \in \Phi\left(\mathcal{W}_{i}\right)} p G\left\|\left(X_{i}, Y_{i},-h\right)\right\|^{-\alpha} \mathbb{1}_{\left\{\Phi\left(\mathcal{W}_{i}\right) \neq \emptyset\right\}}} \geq \tau\right) \\
& \stackrel{(\mathrm{b})}{=} \mathbf{P}_{\Psi}^{0}\left(G \geq I \tau p^{-1}\left(X_{0}^{2}+Y_{0}^{2}+h^{2}\right)^{\frac{\alpha}{2}}\right) \\
& =\mathbf{E}_{X, Y, I}\left[\left.\sum_{i=0}^{m-1} \frac{(-s)^{i}}{i !} \frac{\mathrm{d}^{i}}{\mathrm{~d} s^{i}} e^{-m s / \Omega}\right|_{s=I \tau p^{-1}\left(X^{2}+Y^{2}+h^{2}\right)^{\frac{\alpha}{2}}}\right],
\end{aligned}
$$

where $\mathbf{E}_{X_{0}, Y_{0}, I}$ denotes the expectation w.r.t. the random variables $X_{0}, Y_{0}$ and $I$, where $I$ is the interference seen by the typical UAV at the origin. Given that the location of the access-granted IoT device in the typical window $\left(X_{0}, Y_{0}\right)$ is 


$$
\begin{gathered}
\prod_{i \in \mathbb{Z}}\left(e^{-\lambda w l}+\frac{1-e^{-\lambda w l}}{w l} \int_{i \mu-\frac{w}{2}}^{i \mu+\frac{w}{2}} \int_{-\frac{l}{2}}^{\frac{l}{2}}\left(\frac{1}{1+\frac{s p \Omega m^{-1}}{\left(x^{2}+y^{2}+h^{2}\right)^{\alpha / 2}}}\right) \mathrm{d} y \mathrm{~d} x\right) . \\
\prod_{i \in \mathbb{Z}}^{\neq 0}\left(e^{-\lambda w l}+\frac{1-e^{-\lambda w l}}{w l} \int_{i \mu-\frac{w}{2}}^{i \mu+\frac{w}{2}} \int_{-\frac{l}{2}}^{\frac{l}{2}}\left(\frac{1}{1+s p \Omega m^{-1}\left(x^{2}+y^{2}+h^{2}\right)^{-\frac{\alpha}{2}}}\right) \mathrm{d} y \mathrm{~d} x\right) . \\
\prod_{i \in \mathbb{Z}}\left(e^{-\lambda w l}+\frac{1-e^{-\lambda w l}}{w l} \int_{i \mu-\frac{w}{2}}^{i \mu+\frac{w}{2}} \int_{-\frac{l}{2}}^{\frac{l}{2}} \frac{1}{1+s p\left(x^{2}+y^{2}+h^{2}\right)^{-\frac{\alpha}{2}}} \mathrm{~d} y \mathrm{~d} x\right) .
\end{gathered}
$$

independent of the interference, the coverage probability of the typical UAV is given by

$$
\begin{aligned}
& \frac{\mathbf{P}_{\Psi}^{0}\left(\Phi\left(\mathcal{W}_{0}\right) \neq \emptyset\right)}{w l} \\
& \times \int_{-\frac{w}{2}}^{\frac{w}{2}} \int_{-\frac{l}{2}}^{\frac{l}{2}}\left(\left.\sum_{i=0}^{m-1} \frac{(-s)^{i}}{i !} \frac{\mathrm{d}^{i}}{\mathrm{~d} s^{i}} \mathcal{L}_{I}(s)\right|_{s=\frac{\tau m\left(x^{2}+y^{2}+h^{2}\right)^{\frac{\alpha}{2}}}{p \Omega}}\right) \mathrm{d} y \mathrm{~d} x,
\end{aligned}
$$

where the Laplace transform of the interference $\mathcal{L}_{I}(s)$ is given in Eq. (6).

Remark 3: Since the UAV and IoT point processes are jointly stationary and the IoT point process is mixing, the above coverage expression can be interpreted in an ergodic sense [48]. The coverage probability is also the proportion of time that the typical UAV has an IoT device in its window and successfully decodes its signal.

Example 2: With fading parameters $m=\Omega=1$, the coverage probability is given by Eq. (9).

Fig. 4 illustrates the coverage probability of the typical UAV obtained by formula Eq. (9). Simulation results are presented to validate the accuracy of the derived results. The simulation results and analytical results match. We found that when $\lambda=$ $100 / \mathrm{km}^{2}$, and $\mu=1 \mathrm{~km}$, an increase of the window size decreases the SIR coverage probability of the typical UAV due to the decrease of the desired signal power and the increase of interference. In addition, there exists an upper bound on the coverage probability, which is given by $1-e^{-\lambda w l}$. This value corresponds to the probability that the window of the typical UAV is not empty of IoT devices. In this sense, the coverage probability captures the utilization at UAVs.

\section{Mean Data Rate of the Typical UAV}

This section analyzes the mean UAV data rate and the associated spectral efficiency. Let us consider a fixed $M$-ary modulation with minimum SIR threshold $\tau$. The mean data rate of the typical UAV is then defined by $\log _{2}(M) \mathbf{P}_{\Psi}^{0}(\mathrm{SIR} \geq$ $\tau)$.

Remark 4: For practical reasons, we consider a fixed $M$ ary modulation at the IoT devices transmitters. Under the TDMA scheduling where the transmission channel from the IoT devices to the UAV varies w.r.t. space and time, channelside information is unavailable at the UAVs. Consequently, the fixed modulation with threshold $\tau$ enables reliable uplink transmissions and reduces the chance of transmission failure.
Theorem 3: The mean data rate seen by the typical UAV, $\mathcal{R}$, is given by Eq. 10 .

Proof: The proof immediately follows from Theorem 2

Example 3: With fading parameters $m=\Omega=1$, the typical data rate is given by Eq. (11)

Figs. 5 and 6 plot the mean uplink data rate seen by the typical UAV. The surface in Fig. 5 is non-smooth because of the condition $M=2^{\left\lfloor\log _{2}(1+\tau)\right\rfloor}$. Fig. 6 illustrates the mean data rate of the typical UAV for various SIR thresholds $\tau$. It shows that the analytically derived data rate results exactly match the simulated data rate results. The mean data rate is given by $\log _{2}(1+\tau) \mathbf{P}(\mathrm{SIR} \geq \tau)$, which is the product of an increasing and a decreasing function w.r.t. $\tau$. This explains why, for a given density of IoT devices and UAVs, there exists an optimal SIR threshold $\tau$ that maximizes the data rate seen by the typical UAV. Notice that the SIR threshold may not be as configurable as other network parameters, like, e.g., the scheduling window $w$ because, in practice, the value might be determined by the transmit power of the IoT devices and the bandwidth that these devices use.

Remark 5: Consider an IoT deployment scenario where the chance of having a window with no IoT device is very small; $\mathbf{P}\left(\Phi\left(\mathcal{W}_{0}\right) \neq \emptyset\right) \approx 1$. Such a condition would be met for parameters $\lambda=500 / \mathrm{km}^{2}, w=100 \mathrm{~m}$, and $l=100 \mathrm{~m}$. Conditioning on the fact that the typical window $\mathcal{W}_{0}$ is not empty of IoT devices, the coverage probability of the typical $\mathrm{UAV}, \mathbf{P}_{\Psi}^{0}\left(\operatorname{SIR} \geq \tau \mid \Phi\left(\mathcal{W}_{0}\right) \neq \emptyset\right)$, is approximately given by

$$
\frac{1}{w l} \int_{-\frac{w}{2}}^{\frac{w}{2}} \int_{-\frac{l}{2}}^{\frac{l}{2}} \underbrace{\sum_{i=0}^{m-1} \frac{(-s)^{i}}{i !} \frac{\mathrm{d}^{i}}{\mathrm{~d} s^{i}} \mathcal{L}_{I}(s)}_{s=\frac{\tau m\left(x^{2}+y^{2}+h^{2}\right)^{\frac{\alpha}{2}}}{p \Omega}} \mathrm{d} y \mathrm{~d} x .
$$

Conditionally on the fact that its window is not empty of IoT devices, the data rate $\overline{\mathcal{R}}$ of the typical UAV is now of the form

$$
\frac{\log _{2}(M)}{w l} \int_{-\frac{w}{2}}^{\frac{w}{2}} \int_{-\frac{l}{2}}^{\frac{l}{2}} \underbrace{\sum_{i=0}^{m-1} \frac{(-s)^{i}}{i !} \frac{\mathrm{d}^{i}}{\mathrm{~d} s^{i}} \mathcal{L}_{I}(s)}_{s=\frac{\tau m\left(x^{2}+y^{2}+h^{2}\right)^{\frac{\alpha}{2}}}{p \Omega}} \mathrm{d} y \mathrm{~d} x .
$$

The above quantity in Eq. 13 should not be confused with $\mathcal{R}$, the data rate of the typical UAV in Eq. (10). When the density of IoT devices is high enough to ensure that the window is rarely empty of IoT device, $\mathcal{R} \approx \overline{\mathcal{R}}$. 


$$
\frac{1-e^{-\lambda w l}}{w l} \int_{-\frac{w}{2}}^{\frac{w}{2}} \int_{-\frac{l}{2}}^{\frac{l}{2}} \prod_{k \in \mathbb{Z} \backslash 0}\left(e^{-\lambda w l}+\frac{1-e^{-\lambda w l}}{w l} \int_{k \mu-\frac{w}{2}}^{k \mu+\frac{w}{2}} \int_{-\frac{l}{2}}^{\frac{l}{2}} \frac{\mathrm{d} u \mathrm{~d} v}{1+\frac{\tau\left(x^{2}+y^{2}+h^{2}\right)^{\frac{\alpha}{2}}}{\left(u^{2}+v^{2}+h^{2}\right)^{\frac{\alpha}{2}}}}\right) \mathrm{d} y \mathrm{~d} x .
$$

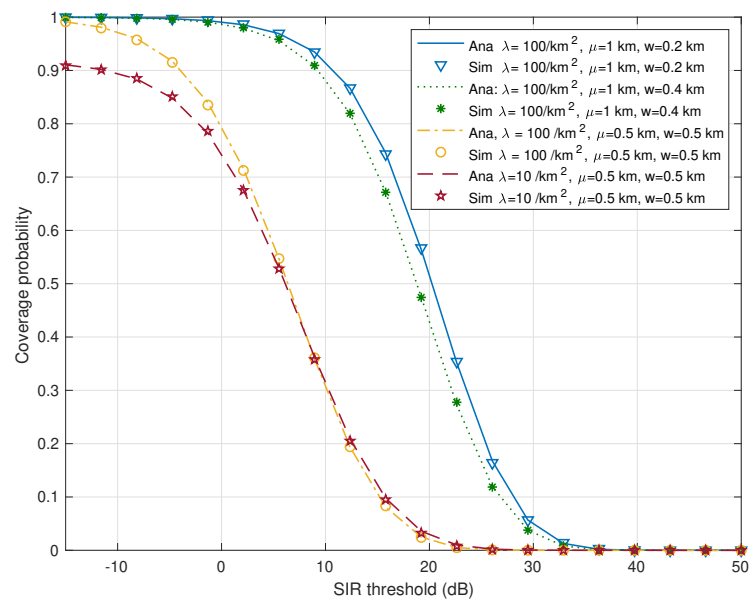

Fig. 4. The coverage probability of the typical UAV. We consider $\alpha=4$, $h=0.2 \mathrm{~km}, l=0.5 \mathrm{~km}$, and $\Omega=m=1$.

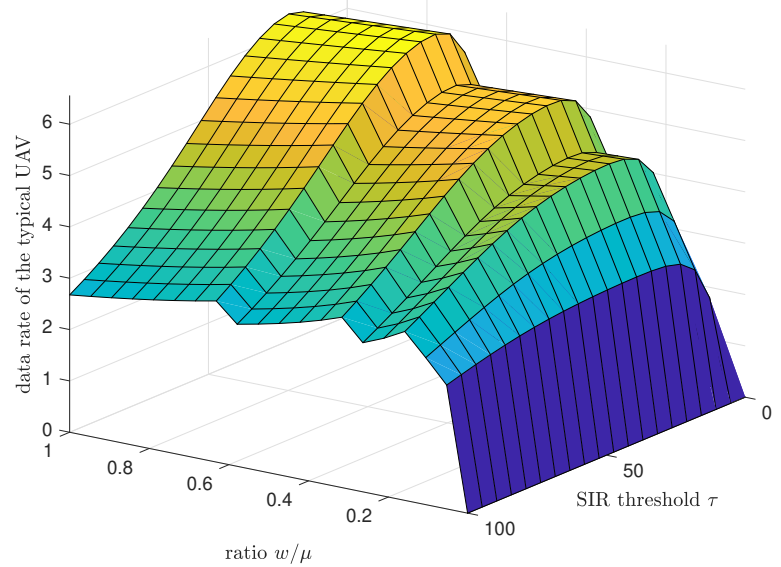

Fig. 5. The data rate at the typical UAV where a typical $M$-ary modulation: $M=2^{\left\lfloor\log _{2}(1+\tau)\right\rfloor}, \Omega=m=1$. Note that the $z$-axis is in bit/sec/Hz.

\section{Performance From the Iot Devices' PERSPECTIVE}

This section focuses on the network performance as seen by a typical IoT device. Specifically, we derive the total amount of data transmitted, i.e., harvested from the typical IoT device to a UAV, while the former is inside the activation window of the latter. This is evaluated under the Palm distribution of the IoT point process,

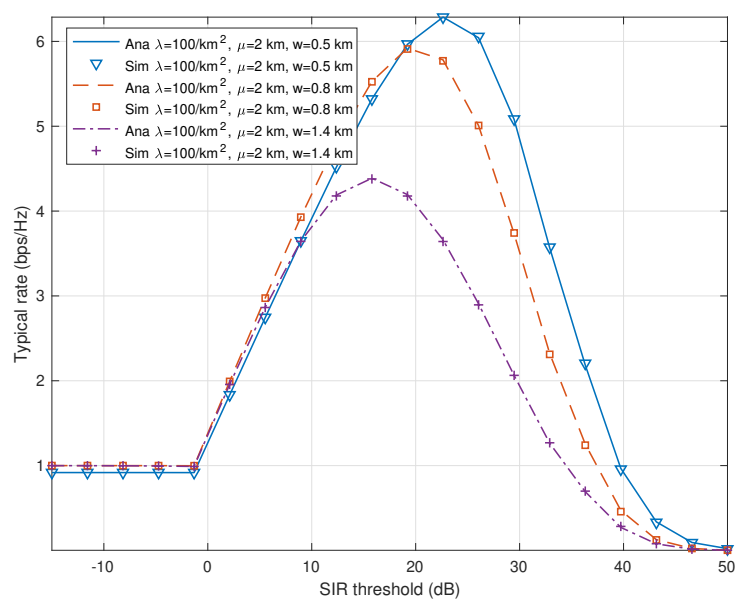

Fig. 6. The data rate seen by the typical UAV. We consider $\alpha=4, h=0.2$ $\mathrm{km}, l=0.5 \mathrm{~km}$ and $m=\Omega=1$. The SIR threshold on the $x$-axis indicates the minimum SIR value for which uplink communications are reliable.

\section{A. Mean Amount of Data Transmitted from a Typical IoT Device}

The coverage probability and rate analysis in the previous section capture the instantaneous network performance at a UAV. This subsection is focused on the evaluation of the meant amount of data transmitted from the typical IoT device per UAV passage.

Here are a few preliminary observations and definitions before stating the main theorem. We recall that in each activation window, there is a random (Poisson) number of IoT devices present at each time slot and that the processing by the UAV is shared between these IoT devices, based on TDMA. As the UAV moves, the TDMA scheme involves a different set of devices. Consequently, in order to compute the mean amount of data uploaded from a typical IoT device to a UAV while it is inside the activation window of the UAV, one should consider the evolution of the network geometry w.r.t. time. Typicality, above and in the theorem below, is again defined in terms of the Palm probability of the IoT point process.

Theorem 4: The mean amount of data $\mathcal{D}$ harvested from the typical IoT device per UAV passage is given by Eq. [12].

Proof: The $y$ coordinates of the points of the IoT point process $\Phi$ of intensity $\lambda$ on the strip can be considered as i.i.d. marks of a linear Poisson point process $\tilde{\Phi}$ with intensity $\lambda l$ on the $x$-axis. Under the Palm distribution of the IoT point process, the typical IoT device is located at the origin with its mark $Y \sim$ Uniform $\left[-\frac{l}{2}, \frac{l}{2}\right]$. The total amount of data transmitted from the typical IoT device to its serving UAV 


$$
\begin{gathered}
\frac{\log _{2}(M)\left(1-e^{-\lambda w l}\right)}{w l} \int_{-\frac{w}{2}}^{\frac{w}{2}} \int_{-\frac{l}{2}}^{\frac{l}{2}}\left(\left.\sum_{i=0}^{m-1} \frac{(-s)^{i}}{i !} \frac{\mathrm{d}^{i}}{\mathrm{~d} s^{i}} \mathcal{L}_{I}(s)\right|_{s=\frac{\tau m\left(x^{2}+y^{2}+h^{2}\right)^{\frac{\alpha}{2}}}{p \Omega}}\right) \mathrm{d} y \mathrm{~d} x . \\
\frac{\log _{2}(M)\left(1-e^{-\lambda w l)}\right.}{w l} \int_{-\frac{w}{2}}^{\frac{w}{2}} \int_{-\frac{l}{2}}^{\frac{l}{2}} \prod_{k \in \mathbb{Z} \backslash 0}\left(e^{-\lambda w l}+\frac{1-e^{-\lambda w l}}{w l} \int_{k \mu-\frac{w}{2}}^{k \mu+\frac{w}{2}} \int_{-\frac{l}{2}}^{\frac{l}{2}} \frac{\mathrm{d} u \mathrm{~d} v}{1+\frac{\tau\left(x^{2}+y^{2}+h^{2}\right)^{\frac{\alpha}{2}}}{\left(u^{2}+v^{2}+h^{2}\right)^{\frac{\alpha}{2}}}}\right) \mathrm{d} y \mathrm{~d} x . \\
\left.\frac{\left(1-e^{-\lambda w l}\right) \log _{2}(M)}{\lambda w l^{2}} \frac{1}{v} \int_{-\frac{w}{2}}^{\frac{w}{2}} \int_{-\frac{l}{2}}^{\frac{l}{2}} \sum_{i=0}^{m-1} \frac{(-s)^{i}}{i !} \frac{\mathrm{d}^{i}}{\mathrm{~d} s^{i}} \mathcal{L}_{I}(s)\right|_{s=\frac{\tau m\left(x^{2}+y^{2}+h^{2}\right)^{\frac{\alpha}{2}}}{p \Omega}} \mathrm{d} y \mathrm{~d} x .
\end{gathered}
$$

is the given by

$$
\mathcal{D}=\mathbf{E}_{\tilde{\Phi}}^{0}\left[\sum_{k=-\left\lfloor\frac{w}{2 v T_{s}}\right\rfloor}^{\left\lfloor\frac{w}{2 v T_{s}}\right\rfloor} \mathcal{R}\left(Y, k T_{s}, \tilde{\Phi}\right)\right],
$$

where $\mathbf{E}_{\tilde{\Phi}}^{0}$ denotes the Palm expectation w.r.t. $\tilde{\Phi}$, $\mathcal{R}\left(Y, k T_{s}, \Phi, \Psi(k)\right)$ is the amount of data that the typical IoT device, with its mark randomly distributed between $-\frac{l}{2}$ and $\frac{l}{2}$, transmits to its serving UAV at time slot $k$. The rate $\mathcal{R}\left(Y, k T_{s}, \tilde{\Phi}\right)$ is given by

$$
\begin{aligned}
\mathcal{R}\left(Y, k T_{s}, \tilde{\Phi}\right) & =T_{s} \log _{2}(M) \\
& \times \mathbb{1}_{\left\{\operatorname{SIR}_{(0, Y, 0) \rightarrow\left(-v k T_{s}, 0, h\right) \geq \tau}\right\}} \\
& \times \mathbb{1}_{\{(0, Y) \text { is selected to transmit at slot } k\}} .
\end{aligned}
$$

The above formula is justified when we assume that $T_{s}$ is sufficiently small, so that the relative positions of $(0, Y)$ and the position of the UAV $\left(-v k T_{s}, 0, h\right)$ do not vary much during the $k$-th time slot. Under this assumption, one can further approximate the summation of Eq. 147 as the following integral:

$$
\mathcal{D} \approx \mathbf{E}_{\tilde{\Phi}}^{0}\left[\int_{-\frac{w}{2 v}}^{\frac{w}{2 v}} \mathcal{R}(Y, t, \tilde{\Phi}) \mathrm{d} t\right],
$$

where the continuous rate $\mathcal{R}(Y, t, \tilde{\Phi})$ is now given by

$$
\begin{aligned}
& \log _{2}(M) \mathbb{1}_{\left\{\operatorname{SIR}_{(0, Y, 0) \rightarrow(v t, 0, h) \geq \tau}\right\}} \mathbb{1}_{\{Y \text { is selected to transmit at time } t\}} \\
& =\log _{2}(M) \sum_{k=1}^{\infty}\left(\mathbb{1}_{\left\{\operatorname{SIR}_{(0, Y, 0) \rightarrow(v t, 0, h) \geq \tau}\right\}}\right. \\
& \left.\mathbb{1}_{\left\{Y \text { is selected out of } k \mid \tilde{\Phi}\left(\mathcal{W}_{v t}\right)=k\right\}} \mathbb{1}_{\left\{\tilde{\Phi}\left(\mathcal{W}_{v t}\right)=k\right\}}\right) \\
& =\log _{2}(M) \sum_{k=1}^{\infty}\left(\mathbb{1}_{\left\{Y \text { is selected out of } k \mid \tilde{\Phi}\left(\mathcal{W}_{v t}\right)=k\right\}}\right. \\
& \mathbb{1}_{\left\{\operatorname{SIR}_{(0, Y, 0) \rightarrow(v t, 0, h) \geq \tau\}} \mathbb{1}_{\left\{\tilde{\Phi}\left(\mathcal{W}_{v t}\right)=k\right\}}\right),}
\end{aligned}
$$

where we use the following facts: 1) the rate from the typical IoT to the typical UAV is positive if and only if it is selected to transmit; 2) one can write that $\mathbb{1}_{A}=\sum_{k} \mathbb{1}_{A \cap B_{k}} \mathbb{1}_{B_{k}}$ where $B_{k}$ is any partition of the sample space; and 3) conditionally on the fact that the number of the IoT device in the activation window centered at $v t, \mathcal{W}_{v t}=\left[v t-\frac{w}{2}, v t+\frac{w}{2}\right] \times\left[-\frac{l}{2}, \frac{l}{2}\right]$, is $k$, the probability for the typical IoT device to be selected to transmit is $1 / k$.

As a result, combining Eqs. (16) and (17), we obtain the Eq. (18) for the amount of data transmitted from the typical IoT. To obtain (a) in Eq. (18), we use Fubini's theorem. To obtain (b) in Eq. (18), we use the fact that $Y$ is an independent mark of $\tilde{\Phi}$, where $Y \sim$ Uniform $\left[-\frac{l}{2}, \frac{l}{2}\right]$ and the fact that the probability that $Y$ is selected out of $k$ IoT devices is equal to $1 / k$. To derive (c) in Eq. (18), we use the independence of the numbers of points of a Poisson point process in disjoint sets, where the first term inside the expectation corresponds to the event that the SIR is greater than $\tau$ and the second term corresponds to the event that the window of serving UAV has $k$ points.

Furthermore, the first integrand of Eq. (18) is given by

$$
\begin{aligned}
& \mathbf{E}_{\tilde{\Phi}}^{0}\left(\mathbb{1}_{\left.\operatorname{SIR}_{(0, y, 0) \rightarrow(v t, 0, h)} \geq \tau\right)}\right) \\
& =\mathbf{P}_{\tilde{\Phi}}^{0}\left(\operatorname{SIR}_{(0, y, 0) \rightarrow(v t, 0, h)} \geq \tau\right) \\
& =\mathbf{P}_{\tilde{\Phi}}^{0}\left(\frac{p G\|(-v t, y,-h)\|^{-\alpha}}{\sum_{i \in \mathbb{Z}}^{\neq 0} p G_{i}\left\|\left(X_{i}-v t, Y_{i},-h\right)\right\|^{-\alpha} \mathbb{1}_{\left\{\tilde{\Phi}\left(\mathcal{W}_{i}\right) \neq \emptyset\right\}}} \geq \tau\right) \\
& =\mathbf{P}\left(G \geq \tau p^{-1} I\left(v^{2} t^{2}+y^{2}+h^{2}\right)^{\frac{\alpha}{2}}\right) \\
& =\left.\sum_{i=0}^{m-1} \frac{(-s)^{i}}{i !} \frac{\mathrm{d}^{i}}{\mathrm{~d} s^{i}} \mathcal{L}_{I}(s)\right|_{s=\frac{\tau m\left(v^{2} t^{2}+y^{2}+h^{2}\right)^{\frac{\alpha}{2}}}{p \Omega}}
\end{aligned}
$$

where $\left(X_{i}, Y_{i}, 0\right)$ denotes the location of the IoT device selected to transmit in the window $\mathcal{W}_{i}:=\left[i \mu+v t-\frac{w}{2}, i \mu+v t+\frac{w}{2}\right] \times\left[-\frac{l}{2}, \frac{l}{2}\right]$ and $\mathcal{L}_{I}(s)$ is the Laplace transform of the interference derived in Theorem 1 .

Using Slivnyak's theorem, the second integrand of Eq. 18 is given by

$$
\begin{aligned}
& \mathbf{E}_{\tilde{\Phi}}^{0}\left[\mathbb{1}_{\tilde{\Phi}\left(\mathcal{W}_{v t}\right)=k}\right] \\
& =\mathbf{E}\left[\mathbb{1}_{\tilde{\Phi}+\delta_{0}\left(\mathcal{W}_{v t}\right)=k}\right] \\
& =\mathbf{E}\left[\mathbb{1}_{\tilde{\Phi}\left(\mathcal{W}_{v t}\right)=k-1}\right] \\
& =\mathbf{P}\left(\tilde{\Phi}\left(\mathcal{W}_{v t}\right)=k-1\right)=\frac{e^{-\lambda w l}(\lambda w l)^{k-1}}{(k-1) !}
\end{aligned}
$$

where we use the fact that intensity of $\tilde{\Phi}$ is $\lambda l$. Eq. (20) holds for $k=1,2,3, \ldots$ with $0 !=1$. As a result, by combining Eq. 


$$
\begin{aligned}
\mathcal{D} & =\log _{2}(M) \mathbf{E}_{\tilde{\Phi}}^{0}\left[\int_{-\frac{w}{2 v}}^{\frac{w}{2 v}}\left(\sum_{k=1}^{\infty} \mathbb{1}_{\left\{Y \text { is selected out of } k \mid \tilde{\Phi}\left(\mathcal{W}_{v t}\right)=k\right\}} \mathbb{1}_{\operatorname{SIR}_{(0, Y, 0) \rightarrow(v t, 0, h)} \geq \tau} \mathbb{1}_{\tilde{\Phi}\left(\mathcal{W}_{v t}\right)=k}\right) \mathrm{d} t\right] \\
& \stackrel{(\mathrm{a})}{=} \log _{2}(M) \int_{-\frac{w}{2 v}}^{\frac{w}{2 v}} \mathbf{E}_{\tilde{\Phi}}^{0}\left[\sum_{k=1}^{\infty} \mathbb{1}_{\left\{Y \text { is selected out of } k \mid \tilde{\Phi}\left(\mathcal{W}_{v t}\right)=k\right\}} \mathbb{1}_{\operatorname{SIR}_{(0, Y, 0) \rightarrow(v t, 0, h)} \geq \tau} \mathbb{1}_{\left.\tilde{\Phi}\left(\mathcal{W}_{v t}\right)=k\right]} \mathrm{d} t\right. \\
& \stackrel{(\mathrm{b})}{=} \log _{2}(M) \int_{-\frac{w}{2 v}}^{\frac{w}{2 v}} \int_{-\frac{l}{2}}^{\frac{l}{2}}\left(\sum _ { k = 1 } ^ { \infty } \frac { 1 } { k } \mathbf { E } _ { \tilde { \Phi } } ^ { 0 } \left[\mathbb{1}_{\operatorname{SIR}_{(0, y, 0) \rightarrow(v t, 0, h)} \geq \tau} \mathbb{1}_{\left.\left.\tilde{\Phi}\left(\mathcal{W}_{v t}\right)=k\right]\right)} \frac{\mathrm{d} y}{l} \mathrm{~d} t\right.\right. \\
& \stackrel{(\mathrm{c})}{=} \log _{2}(M) \int_{-\frac{w}{2 v}}^{\frac{w}{2 v}} \int_{-\frac{l}{2}}^{\frac{l}{2}}\left(\sum _ { k = 1 } ^ { \infty } \frac { 1 } { k } \mathbf { E } _ { \tilde { \Phi } } ^ { 0 } [ \mathbb { 1 } _ { \operatorname { S I R } _ { ( 0 , y , 0 ) \rightarrow ( v t , 0 , h ) } \geq \tau } ] \mathbf { E } _ { \Phi } ^ { 0 } \left[\mathbb{1}_{\left.\left.\tilde{\Phi}\left(\mathcal{W}_{v t}\right)=k\right]\right)} \frac{\mathrm{d} y}{l} \mathrm{~d} t .\right.\right.
\end{aligned}
$$

(18), (19), and (20), we have $\mathcal{D}$

$$
\begin{aligned}
& \log _{2}(M) \int_{-\frac{w}{2 v}}^{\frac{w}{2 v}} \int_{-\frac{l}{2}}^{\frac{l}{2}} \underbrace{\sum_{i=0}^{m-1} \frac{(-s)^{i}}{i !} \frac{\mathrm{d}^{i}}{\mathrm{~d} s^{i}} \mathcal{L}_{I}(s)}_{s=\frac{\tau m\left(v^{2} t^{2}+y^{2}+h^{2}\right)^{\frac{\alpha}{2}}}{p \Omega}} \\
& \left.\left(\sum_{k=1}^{\infty} \frac{e^{-\lambda w l}(\lambda w l)^{k-1}}{k(k-1) !}\right)\right) \frac{\mathrm{d} y}{l} \mathrm{~d} t \\
& =\frac{\log _{2}(M)}{l \cdot \lambda w l} \int_{-\frac{w}{2 v}}^{\frac{w}{2 v}} \int_{-\frac{l}{2}}^{\frac{l}{2}} \underbrace{\sum_{i=0}^{m-1} \frac{(-s)^{i}}{i !} \frac{\mathrm{d}^{i}}{\mathrm{~d} s^{i}} \mathcal{L}_{I}(s)}_{s=\frac{\tau m\left(v^{2} t^{2}+y^{2}+h^{2}\right)^{\frac{\alpha}{2}}}{p \Omega}} \\
& \left(\sum_{k=1}^{\infty} \frac{e^{-\lambda w l}(\lambda w l)^{k}}{k !}\right) \mathrm{d} y \mathrm{~d} t \\
& =\frac{\left(1-e^{-\lambda w l}\right) \log _{2}(M)}{\lambda w l^{2}} \frac{1}{v} \\
& \times \int_{-\frac{w}{2}}^{\frac{w}{2}} \int_{-\frac{l}{2}}^{\frac{l}{2}} \underbrace{\sum_{i=0}^{m-1} \frac{(-s)^{i}}{i !} \frac{\mathrm{d}^{i}}{\mathrm{~d} s^{i}} \mathcal{L}_{I}(s)}_{s=\frac{\tau m\left(x^{2}+y^{2}+h^{2}\right)^{\frac{\alpha}{2}}}{p \Omega}} \mathrm{d} y \mathrm{~d} x .
\end{aligned}
$$

This completes the proof.

Example 4: With parameters $m=\Omega=1$, the mean amount of harvested data is given by Eq. 21.

Here are a few observations on Theorem 4 1) $\mathcal{D}$ is inversely proportional to the speed $v ; \mathcal{D} \propto v^{-1}$. If UAVs move faster, each UAV provides a shorter duration of active time for each IoT device, and consequently, the average amount of harvested data from each device decreases. Nevertheless, note that when UAV move faster, each IoT device is scheduled by a larger number of UAVs per unit time on average. Specifically, the average amount of harvested data per unit time is given by $\frac{\mathcal{D} v}{\mu}$ since $v / \mu$ UAVs pass each IoT device per unit time. 2) $\mathcal{D}$ is monotonically increasing w.r.t. $\mu$. As the distance between UAVs increases, the interference power decreases, and thus the coverage and rate increase. Fig. 7 illustrates the evolution of the mean amount of data transmitted from the typical IoT device to a UAV in function of $\tau$ and $w / \mu$. As mentioned in the system model, the size $w$ of the scheduling window is one of the key control parameters. The impact of this parameter on network performance is discussed in the next section.

Remark 6: Both the size of the coverage window and the distance between UAVs could be scaled in order to adapt the density of serving IoT devices.

\section{DiscusSiOn AND EXTENSIONS}

\section{A. Mean Rate Formula and Mass Transport}

In this subsection, we connect the mean data rate of the typical UAV, $\mathcal{R}$, given in Section III, and the mean amount of data transmitted from the typical IoT device, $\mathcal{D}$, given in Section IV by establishing a general relationship between them.

Theorem 5: When the density of IoT devices is high, the mean amount of data harvested from the typical IoT device per UAV passage, $\mathcal{D}$, is linked to the mean rate $\mathcal{R}$ of the typical UAV by the relation

$$
\mathcal{D} \approx \frac{\mathcal{R}}{\mathcal{K}} \mathcal{T}
$$

where $\mathcal{K}=\lambda w l$ is the mean number of IoT devices in each window and $\mathcal{T}=w / v$ is the mean duration the typical IoT device is in the activation window of a given UAV.

Proof: When the density of IoT device is high enough, $1-e^{-\lambda w l} \approx 1$. Then, using Eqs. 10 , and $[12$, we can write

$$
\mathcal{D} \approx \frac{1}{\lambda w l} \frac{w}{v} \mathcal{R}=\frac{\mathcal{R}}{\mathcal{K}} \mathcal{T}
$$

This completes the proof.

Remark 7: In Appendix A, the above mean formula is proved again based on the mass transport principle [50] on a unimodular weighted graph. In the graph, UAVs and the activated IoT devices are vertices of the graph and links from the IoT devices to UAVs are the edges of the graph. This shows that the above relationship holds mainly due to the following two key reasons: 1) the IoT and UAV point processes are jointly stationary and 2) only one IoT device per window is scheduled to transmit and IoT devices are scheduled for the same amount of time, on average. Consequently, the same linear relationship will hold for other and more general network scenarios (e.g., other than Poisson but stationary IoT device point processes, other than Nakagami fading models, etc.). 


$$
\frac{\left(1-e^{-\lambda w l}\right) \log _{2}(M)}{v \lambda w l^{2}} \int_{-\frac{w}{2}}^{\frac{w}{2}} \int_{-\frac{l}{2}}^{\frac{l}{2}} \prod_{k \in \mathbb{Z} \backslash 0}\left(e^{-\lambda w l}+\frac{1-e^{-\lambda w l}}{w l} \int_{k \mu-\frac{w}{2}}^{k \mu+\frac{w}{2}} \int_{-\frac{l}{2}}^{\frac{l}{2}} \frac{\mathrm{d} v \mathrm{~d} u}{1+\frac{\tau\left(x^{2}+y^{2}+h^{2}\right)^{\frac{\alpha}{2}}}{\left(u^{2}+v^{2}+h^{2}\right)^{\frac{\alpha}{2}}}}\right) \mathrm{d} y \mathrm{~d} x .
$$

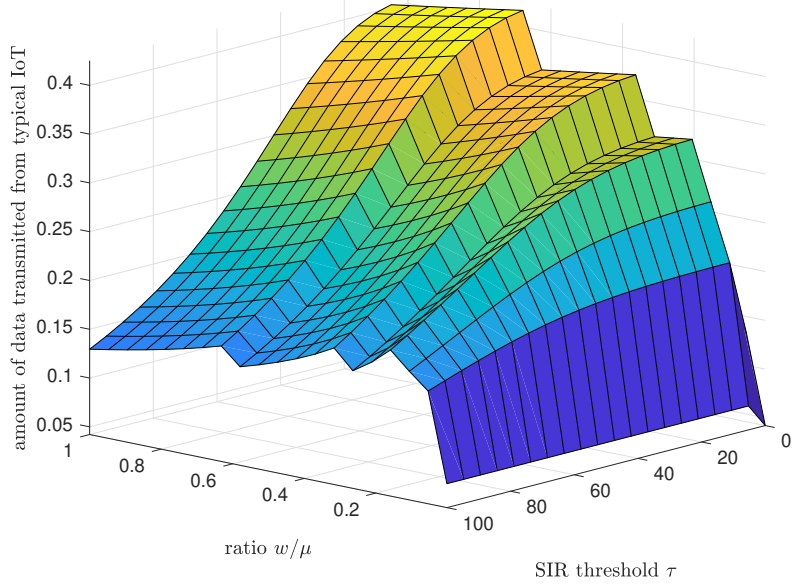

Fig. 7. The amount of data transmitted from the typical IoT device to its serving UAV. We consider $\alpha=3.5, h=0.2 \mathrm{~km}, \mu=2 \mathrm{~km}, l=0.5 \mathrm{~km}$, $v=30 \mathrm{~m} / \mathrm{sec}$, Nakagami fading parameters $\Omega=m=1$, and IoT density $\lambda=1000 / \mathrm{km}^{2}$. Note that the $z$-axis is in $\mathrm{bit} / \mathrm{Hz}$.

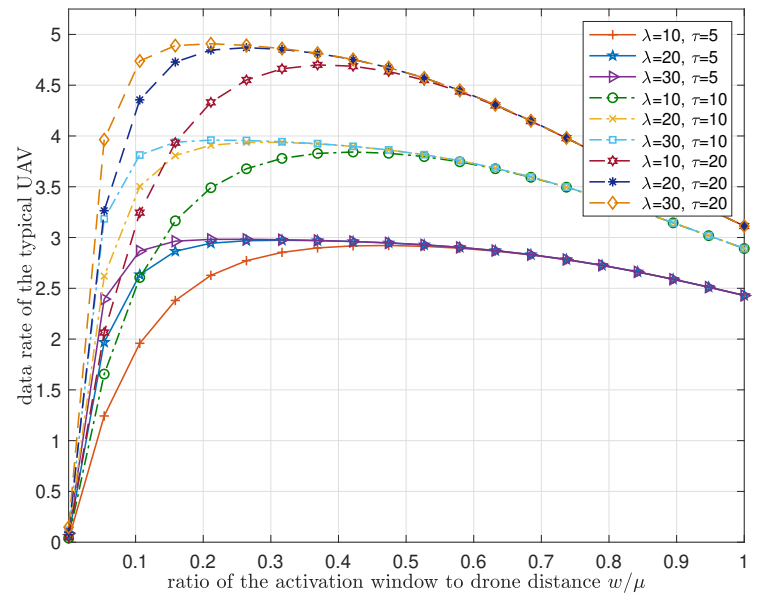

Fig. 8. Illustration of the data rates at the typical UAV. The maximum size of activation window is equal to $\mu=2 \mathrm{~km}$ We consider $\alpha=4, h=0.2$ $\mathrm{km}$, Nakagami fading parameters $m=\Omega=1$, and the modulation rate $M=2^{\left\lfloor\log _{2}(1+\tau)\right\rfloor}$.

\section{B. Performance Optimization w.r.t. Activation Window}

The activation windows determine the area where IoT devices are scheduled for uplink transmissions. In this paper, these windows are modeled by a collection of rectangles on the plane. The length of each window $w$, is a control parameter that should be configured to achieve the best network performance. We recall that we assume that the distance between UAVs is larger than the length of the activation $(\mu>w)$. We show below that $w$ is one of the key optimization parameters of the proposed motion-based harvesting architecture.

Let us discuss the impact of the activation windows on the network performance. If $w$ is too big, i.e., $w \approx \mu$, the key advantage of the proposed architecture vanishes as the idea is to provide universal coverage with a number of UAVs smaller than the number of static base stations that would cover the whole plane. In addition, a larger window leads to a larger number of IoT devices scheduled for the uplink transmissions in the network. Consequently, the average distance from IoT devices to their corresponding UAVs also increases, as well as the interference at any UAV. On the other hand, if the window $w$ is too small, it is very likely that activation windows are empty of IoT devices. Therefore, the upload data rate becomes negligible. Fig. 8 illustrates the dependency of the mean data rate of the typical UAV $\mathcal{R}$ w.r.t. $w$, normalized by the inter-UAV distance. In this figure, we use the parameters $\mu=2 \mathrm{~km}, h=0.25 \mathrm{~km}$ and $\alpha=4$. When $w=0 \mathrm{~km}$, we have $\mathcal{R} \approx 0$. For smaller values of the window size, the data rate increases as the window size increases. For larger values, the data rate decreases as the window size increases. There is a unique value of $w$ where the data rate achieves its maximum. For densities $\lambda=10,20$, and $30 / \mathrm{km}^{2}$, the corresponding optimum window sizes are roughly $0.2 \mu, 0.3 \mu$, and $0.4 \mu$. The figure clearly demonstrates that the optimal size of activation window $w^{\star}$ depends on the density $\lambda$. Generally speaking, for a higher density of IoT devices, a smaller activation window is required to optimize the data rate. The optimal size of window can be numerically found by Eq. (24) where the constant $\log _{2}(1+M)$ is removed from $\mathcal{R}$ for the moment because it cannot change the optimal window size. Solving the above optimization exactly, e.g., KKT condition [51], is beyond of the scope of this paper.

\section{Interference-limited Architecture}

In the coverage analysis, the thermal noise at UAVs is ignored. The major difference the proposed network with the conventional UAV networks is that the proposed data harvesting architecture is based on a fleet of UAVs in a single line (or in parallel lines for the 2-D case that will be provided shortly). Thus, the activation windows of UAVs create a nonnegligible amount of interference at the typical UAV and the proposed network is interference-limited. Table II describes the network parameters used for system-level simulations of the proposed network. In Fig. 9, the SINR and SIR coverage probabilities are illustrated. Each SINR and corresponding SIR are almost identical, which indicates that the noise power at the typical UAV is very small compared to the interference power at the typical UAV for the given parameters. In Fig. 10 the Laplace transforms of the interference and noise power are illustrated. The Laplace transform of the interference plus thermal noise is given by Eq. 25] where $N_{0}$ denotes thermal 


$$
\begin{gathered}
\underset{0 \leq w \leq \mu}{\arg \max } \frac{\left(1-e^{-\lambda w l}\right)}{w l} \int_{-\frac{w}{2}}^{\frac{w}{2}} \int_{-\frac{l}{2}}^{\frac{l}{2}} \prod_{k}^{\mathbb{Z} \backslash 0}\left(e^{-\lambda w l}+\frac{1-e^{-\lambda w l}}{w l} \int_{k \mu-\frac{w}{2}}^{k \mu+\frac{w}{2}} \int_{-\frac{l}{2}}^{\frac{l}{2}} \frac{\mathrm{d} v \mathrm{~d} u}{\left(1+\frac{\Omega \tau\left(x^{2}+y^{2}+h^{2}\right)^{\frac{\alpha}{2}}}{m\left(u^{2}+v^{2}+h^{2}\right)^{\frac{\alpha}{2}}}\right)^{m}}\right) \mathrm{d} y \mathrm{~d} x . \\
e^{-s N_{0}} \prod_{i \in \mathbb{Z}}\left(e^{-\lambda w l}+\frac{1-e^{-\lambda w l}}{w l} \int_{i \mu-\frac{w}{2}}^{i \mu+\frac{w}{2}} \int_{-\frac{l}{2}}^{\frac{l}{2}}\left(\frac{1}{1+\frac{s p \Omega m^{-1}}{\left(x^{2}+y^{2}+h^{2}\right)^{\alpha / 2}}}\right) \mathrm{d} y \mathrm{~d} x\right) .
\end{gathered}
$$

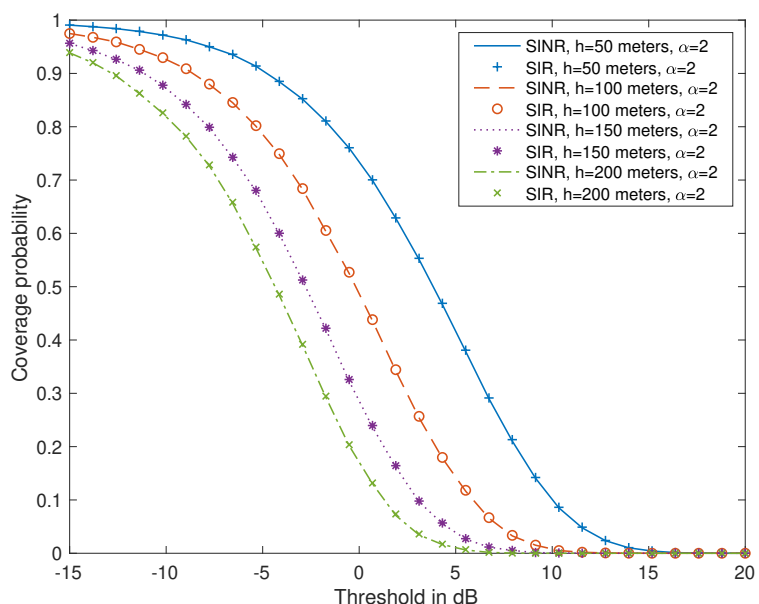

Fig. 9. The simulated probability that the typical SINR and SNR is greater than some threshold $\tau$. I consider $p=23 \mathrm{dBm}, \alpha=2, w, l=$ 100 meters, $\mu=200$ meters, $\sigma^{2}=-104 \mathrm{dBm}$.

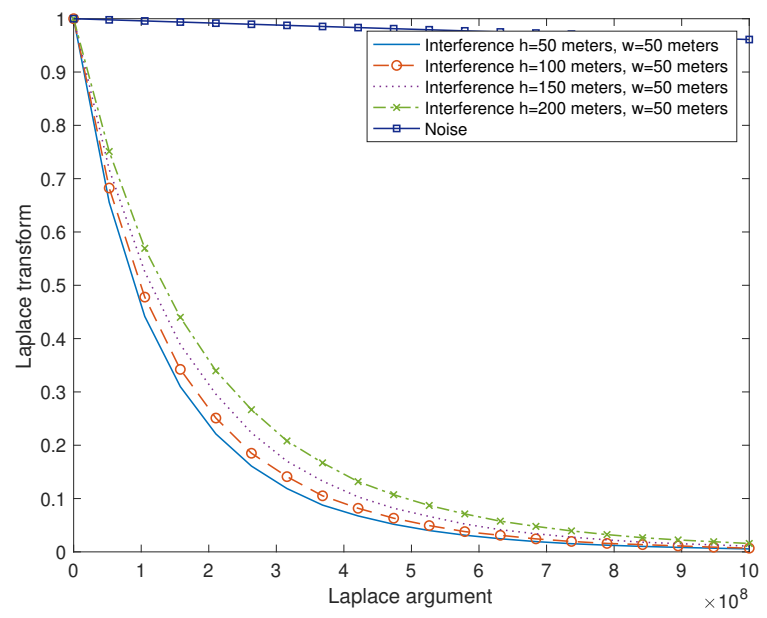

Fig. 10. Illustration of Laplace transform of the interference and noise. We consider $p=23 \mathrm{dBm}, w, l=100$ meters, $\mu=200$ meters, $\sigma^{2}=-104$ $\mathrm{dBm}$.

noise. Note the Laplace transform of the interference is less than the Laplace transform of the noise power, which implies that the interference power is greater than the noise power for the given parameters.
TABLE II

SIMULATION PARAMETERS

\begin{tabular}{|c|c|}
\hline Bandwidth & $10 \mathrm{MHz}$ \\
\hline Density of IoT devices & $10^{5} / \mathrm{km}^{2}$ \\
\hline Transmit power & $23 \mathrm{dBm}$ \\
\hline Noise power density & $-174 \mathrm{dBm} / \mathrm{Hz}$ \\
\hline UAV Altitude & $\{50,100,150,200\}$ meters \\
\hline Carrier frequency & $1 \mathrm{GHz}$ \\
\hline Path loss exponent & 2 \\
\hline Window dimension & $\left\{50 \times 100 \mathrm{~m}^{2}, 100 \times 100 \mathrm{~m}^{2}\right\}$ \\
\hline Inter-UAV distance & 200 meters \\
\hline
\end{tabular}

\section{Two-Dimensional Extensions}

The linear model discussed so far was motivated by applications where UAVs hover or travel, e.g., over a street in a coordinated way to collect data from roadside units. As discussed in the motivation section, for other applications, there is a need for 2-D UAV networks covering in an intermittent but universal way the whole Euclidean plane. In the 2-D extension discussed in this subsection, we assume that IoT devices are distributed according to a planar Poisson point process $\hat{\Phi}$ with spatial density $\hat{\lambda}$. There is a collection of parallel linear trajectories with a periodically spaced fleet of UAVs on each. Each UAV fleets moves along its trajectory with a fixed speed $v$. Hence

$$
\hat{\Psi}(t)=\sum_{i, j \in \mathbb{Z}^{2}} \delta_{(i \mu, j \nu, h)+(U, V, 0)+(v t, 0,0)},
$$

where $U \sim$ Uniform $[-\mu / 2, \mu / 2], V \sim$ Uniform $[-\nu / 2, \nu / 2]$, and $\nu$ is the distance between adjacent UAV trajectories. Similar to the strip UAV model, the randomized shift modeled by $U, V$ ensures the stationarity of the 2-D UAV process $\hat{\Psi}(t)$. The activation window is modeled by $w$ by $l$. Note that if the proposed architecture is meant to provide universal coverage, we will have $w \leq \mu$ and $l=\nu$. Fig. 11 illustrates the 2D model with parameters: $\mu=\nu=l=2 \mathrm{~km}$. Note that other (an possibly better) variants can be considered like, e.g., having an hexagonal grid of UAVs rather than the square one considered in the illustration.

The methodology developed for the 1-D case can be extended step by step.

Corollary 2: The Laplace transform of the uplink shot-noise process of the typical UAV in the 2-D model is given by Eq. 27]. Furthermore, the shot-noise process at the typical UAV is time-invariant.

Proof: Under the Palm distribution of the UAV point process $\hat{\Psi}$, the typical UAV exists at $(0,0, h)$. The 2-D 


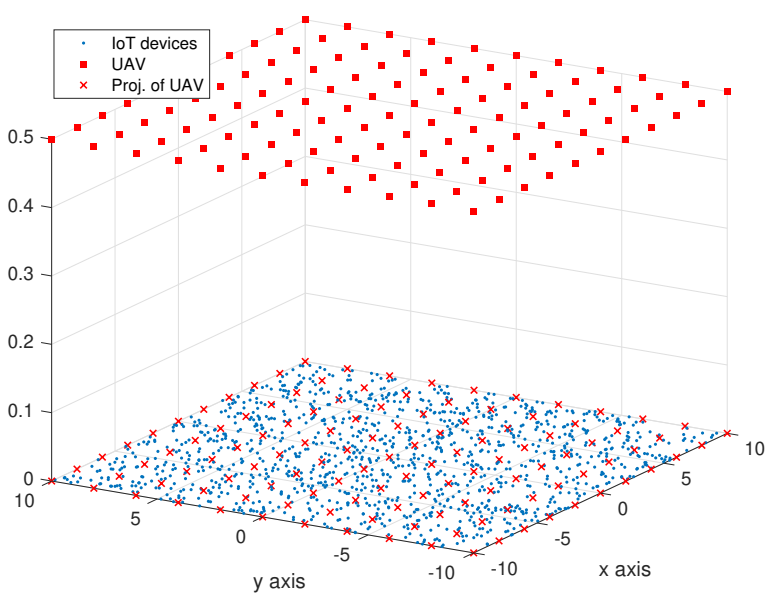

Fig. 11. 2-D extension of the proposed linear model.

activation window $\hat{W}$ is given by

$$
\begin{aligned}
\hat{\mathcal{W}} & =\hat{\mathcal{W}}_{i, j} \\
& =\bigcup_{(i, j) \in \mathbb{Z}^{2}}\left[i \mu-\frac{w}{2}, i \mu+\frac{w}{2}\right] \times\left[j \nu-\frac{l}{2}, j \nu+\frac{l}{2}\right],
\end{aligned}
$$

where $\mu$ is the distance between UAVs on the same trajectory and $\nu$ indicates the distance between UAV trajectories; $w \leq \mu$ and $l \leq \nu$. The shot-noise process at the typical UAV is

$$
N_{2}=\sum_{\left(X_{i, j}, Y_{i, j}\right)} p G\left\|\left(X_{i, j}, Y_{i, j}, 0\right)-(0,0, h)\right\|^{-\alpha} \mathbb{1}_{\left\{\hat{\Phi}\left(\hat{\mathcal{W}}_{i, j}\right) \neq \emptyset\right\}},
$$

where $\left(X_{i, j}, Y_{i, j}\right)$ are the $x, y$ coordinates of the accessgranted IoT devices, if any, in window $\hat{\mathcal{W}}_{i, j}$. Due to the Poisson property, the locations of the access-granted IoT device is uniformly distributed in its corresponding window. Similar to the proof of Theorem 11, we can write $\mathcal{L}_{N_{2}}(s)$ as follows:

$$
\begin{aligned}
& \mathbf{E}_{\hat{\Psi}}^{0}\left[\prod _ { ( X _ { i , j } , Y _ { i , j } ) } ^ { \in \hat { \mathcal { W } } _ { i , j } } \left(\mathbf{P}\left(\hat{\Phi}\left(\hat{\mathcal{W}}_{i, j}\right)=\emptyset\right)\right.\right. \\
& \left.\left.+\mathbf{P}\left(\hat{\Phi}\left(\hat{\mathcal{W}}_{i, j}\right) \neq \emptyset\right) \mathbf{E}\left[e^{-s p G\left\|\left(X_{i, j}, Y_{i, j}, 0\right)-(0,0, h)\right\|^{-\alpha}}\right]\right)\right] \\
& =\mathbf{E}_{\hat{\Psi}}^{0}\left[\prod _ { ( X _ { i , j } , Y _ { i , j } ) } ^ { \in \hat { \mathcal { W } } _ { i , j } } \left(e^{-\hat{\lambda} w l}\right.\right. \\
& \left.\left.+\left(1-e^{-\hat{\lambda} w l}\right) \int_{\operatorname{supp}(G)} e^{-s p g\left(Y_{i, j}^{2}+X_{i, j}^{2}+h^{2}\right)^{-\frac{\alpha}{2}}} f_{G}(g) \mathrm{d} g\right)\right] \\
& \stackrel{(\mathrm{a})}{=} \prod_{(i, j) \in \mathbb{Z}^{2}}\left(e^{-\hat{\lambda} w l}\right. \\
& \left.+\frac{1-e^{-\hat{\lambda} w l}}{w l} \int_{i \mu-\frac{w}{2}}^{i \mu+\frac{w}{2}} \int_{j l-\frac{l}{2}}^{j l+\frac{l}{2}}\left(\frac{1}{1+\frac{s p \Omega}{m\left(x^{2}+y^{2}+h^{2}\right)^{-\frac{\alpha}{2}}}}\right)^{m} \mathrm{~d} y \mathrm{~d} x\right),
\end{aligned}
$$

where we obtain (a) from the fact that the access-granted IoT devices are independent and they are uniformly distributed in each window.
Example 5: The Laplace transform of the interference of the typical UAV is given by Eq. 28.

The Laplace transform of the 2-D shot-noise process can be used to compute the coverage probability and the data rate of the typical UAV.

Corollary 3: The coverage probability of the typical UAV is given by

$$
\frac{1-e^{-\hat{\lambda} w l}}{w l} \int_{-\frac{w}{2}}^{\frac{w}{2}} \int_{-\frac{l}{2}}^{\frac{l}{2}} \underbrace{\sum_{i=0}^{m-1} \frac{(-s)^{i}}{i !} \frac{\mathrm{d}^{i}}{\mathrm{~d} s^{i}} \mathcal{L}_{I_{2}}(s)}_{s \leftarrow \frac{\tau m\left(x^{2}+y^{2}+h^{2}\right)^{\frac{\alpha}{2}}}{p \Omega}} \mathrm{d} y \mathrm{~d} x .
$$

Similarly, the data rate at the typical UAV is given by

$$
\frac{\log _{2}(M)\left(1-e^{-\hat{\lambda} w l}\right)}{w l} \int_{-\frac{w}{2}}^{\frac{w}{2}} \int_{-\frac{l}{2}}^{\frac{l}{2}} \underbrace{\sum_{i=0}^{m-1} \frac{(-s)^{i}}{i !} \frac{\mathrm{d}^{i}}{\mathrm{~d} s^{i}} \mathcal{L}_{I_{2}}(s)}_{s=\frac{\tau m\left(x^{2}+y^{2}+h^{2}\right)^{\frac{\alpha}{2}}}{p \Omega}} \mathrm{d} y \mathrm{~d} x .
$$

In both Eqs, $\mathcal{L}_{I_{2}}(s)$ denotes the Laplace transform of the interference provided in Eq. (28).

Proof: The proof is similar to the proof of Theorem 2.

\section{CONCLUSION}

This paper analyzes the performance of data harvesting architectures based on static IoT devices and a fleet of UAVs with coordinated motion. In the proposed framework, UAVs harvest delay-tolerant data from surface IoT devices, inside their activation windows. Due to the fleet's inherent mobility, the proposed architecture can provide universal coverage for the widely distributed IoT device even when the activation window size is smaller than the instantaneous coverage area. By leveraging the joint stationarity of the spatial model, we derived the coverage probability and the mean data rate of the typical UAV. Similarly, in order to assess the harvesting capability of the proposed architecture, we derived a formula for the mean amount of data uploaded from the typical IoT device to a UAV. This metric was then linked to the data rate of the typical UAV, thanks to the mass transport principle. The key features of the proposed architecture can be summarized as follows:

- Its instantaneous coverage area (activation windows) can be way smaller than the total coverage area at all times, which allows for cost reductions (to the expense of delay).

- It has the potential of providing large-scale universal connectivity with a bounded connectivity delay, both in 1 and 2-D.

- Its key performance metrics are known in closed or integral form.

- Its harvesting capacity can be maximized by selecting some optimal activation window.

\section{APPENDIX A \\ PROOF OF THEOREM 5}

We prove Theorem 5 using the mass transport principle on a stationary graph, defined on jointly stationary point processes. 


$$
\begin{aligned}
& \mathcal{L}_{N_{2}}(s)=\prod_{(i, j) \in \mathbb{Z}^{2} \backslash\{(0,0)\}}\left(e^{-\hat{\lambda} w l}+\frac{1-e^{-\hat{\lambda} w l}}{w l} \int_{i \mu-\frac{w}{2}}^{i \mu+\frac{w}{2}} \int_{j l-\frac{l}{2}}^{j l+\frac{l}{2}} \frac{1}{\left(1+\frac{s p \Omega m^{-1}}{\left(x^{2}+y^{2}+h^{2}\right)^{\frac{\alpha}{2}}}\right)^{m}} \mathrm{~d} y \mathrm{~d} x\right) . \\
& \mathcal{L}_{I_{2}}(s)=\prod_{(i, j) \in \mathbb{Z}^{2}}^{\neq(0,0)}\left(e^{-\hat{\lambda} w l}+\frac{1-e^{-\hat{\lambda} w l}}{w l} \int_{i \mu-\frac{w}{2}}^{i \mu+\frac{w}{2}} \int_{j l-\frac{l}{2}}^{j l+\frac{l}{2}}\left(\frac{1}{1+\frac{s p \Omega}{m\left(x^{2}+y^{2}+h^{2}\right)^{-\frac{\alpha}{2}}}}\right)^{m} \mathrm{~d} y \mathrm{~d} x\right) .
\end{aligned}
$$

Let $\Phi_{1}$ and $\Phi_{2}$ be arbitrary stationary point processes with intensity $\lambda_{1}$ and $\lambda_{2}$, respectively. Let $G$ denote a weighted directed graph with weights equal to the amount of mass transported from the points of $\Phi_{1}$ to the points of $\Phi_{2}$. Assuming that the graph and the weights are factors of $\left(\Phi_{1}, \Phi_{2}\right)$, we have the following mass transport formula:

$$
\frac{\lambda_{1}}{\lambda_{1}+\lambda_{2}} \mathbf{E}_{\Phi_{1}}^{0}\left[G^{+}(0)\right]=\frac{\lambda_{2}}{\lambda_{1}+\lambda_{2}} \mathbf{E}_{\Phi_{2}}^{0}\left[G^{-}(0)\right],
$$

where $G^{+}(0)$ and $G^{-}(0)$ denote the mass out of the origin under $\mathbf{P}_{\Phi_{1}}^{0}$, and the mass toward the origin under $\mathbf{P}_{\Phi_{2}}^{0}$, respectively.

Consider the graph whose vertices are given by $\left(\Psi, \Phi^{\prime}\right)$; the UAV point process $\Psi$ and the corresponding IoT point process $\Phi^{\prime}$ inside the windows of $\Psi$. Note that $\Phi^{\prime} \subset \Phi$ and $\left(\Psi, \Phi^{\prime}\right)$ are joint stationary. Due to the Poisson property, the density of $\Psi$ is $\frac{1}{\mu}$ and the density of $\Phi^{\prime}$ is equal to $\frac{\lambda w l}{\mu}$. Under the TDMA scheduling of UAVs, the weights of edges are the instantaneous data rates from the points of $\Phi^{\prime}$ to the points of $\Psi^{\prime}$. By the mass transport in Eq. [29], we have

$$
\frac{\frac{\lambda w l}{\mu}}{\frac{1}{\mu}+\frac{\lambda w l}{\mu}} \mathcal{R}_{\text {out }}=\frac{\frac{1}{\mu}}{\frac{1}{\mu}+\frac{\lambda w l}{\mu}} \mathcal{R}_{\text {in }},
$$

where $\mathcal{R}_{\text {out }}$ is the instantaneous data rate from the points of $\Phi^{\prime}$ and $\mathcal{R}_{i n}$ is the instantaneous data rate at the points of $\Psi$, namely, the instantaneous data rate seen from the typical UAV. Based on the mass transport principle, we have

$$
\lambda w l \mathcal{R}_{\text {out }}=\mathcal{R}_{\text {in }}=\mathcal{R} .
$$

On other hand, due to the fact that IoT devices are covered by UAVs repeatedly, the amount of data $\mathcal{D}$ transmitted from the typical IoT device per UAV passage is given by

$$
\begin{aligned}
\mathcal{D} & =\mathcal{R}_{\text {out }} \underbrace{\frac{w}{v}}_{\text {(a) }}+0 \cdot \underbrace{\frac{\mu-w}{v}}_{\text {(b) }} \\
& =\mathcal{R}_{\text {out }} \frac{w}{v} \stackrel{\text { (c) }}{=} \mathcal{R}_{\text {in }} \frac{1}{\lambda w l} \frac{w}{v}=\mathcal{R} \frac{\mathcal{T}}{\mathcal{K}},
\end{aligned}
$$

where (a) is the amount of time that the each IoT device is inside each window and (b) is the amount of time that each IoT device is outside of each window. To derive (c), we use Eq. 30. This completes the proof.

\section{REFERENCES}

[1] C. Choi, F. Baccelli, and G. de Veciana, "Analysis of data harvesting by unmanned aerial vehicles," in Proc. IEEE ISIT 2019, July, pp. 121-125.

[2] J. Gubbi, R. Buyya, S. Marusic, and M. Palaniswami, "Internet of things (IoT): A vision, architectural elements, and future directions," Future generation computer systems, vol. 29, no. 7, pp. 1645-1660, July 2013.
[3] L. D. Xu, W. He, and S. Li, "Internet of things in industries: A survey," IEEE Trans. Industrial Informatics, vol. 10, no. 4, pp. 2233-2243, Nov 2014.

[4] A. H. Ngu, M. Gutierrez, V. Metsis, S. Nepal, and Q. Z. Sheng, "IoT middleware: A survey on issues and enabling technologies," IEEE Internet Things J., vol. 4, no. 1, pp. 1-20, Feb 2017.

[5] V. Baiocchi, D. Dominici, and M. Mormile, "UAV application in postseismic environment," International Archives of the Photogrammetry, Remote Sensing and Spatial Information Sciences, vol. 1, p. W2, 2013.

[6] A. Gaur, B. Scotney, G. Parr, and S. McClean, "Smart city architecture and its applications based on iot," Procedia computer science, vol. 52, pp. 1089-1094, 2015.

[7] A. Botta, W. De Donato, V. Persico, and A. Pescapé, "Integration of cloud computing and Internet of things: a survey," Future generation computer systems, vol. 56, pp. 684-700, 2016.

[8] R. Ratasuk, B. Vejlgaard, N. Mangalvedhe, and A. Ghosh, "NB-IoT system for M2M communication," in Proc. IEEE WCNC Workshops 2016, Apr., pp. 428-432.

[9] Y. . E. Wang, X. Lin, A. Adhikary, A. Grovlen, Y. Sui, Y. Blankenship, J. Bergman, and H. S. Razaghi, "A primer on 3GPP narrowband internet of things," IEEE Commun. Mag., vol. 55, no. 3, pp. 117-123, Mar. 2017.

[10] R. S. Sinha, Y. Wei, and S.-H. Hwang, "A survey on LPWA technology: LoRa and NB-IoT," Ict Express, vol. 3, no. 1, pp. 14-21, Mar. 2017.

[11] U. Raza, P. Kulkarni, and M. Sooriyabandara, "Low power wide area networks: An overview," IEEE Commun. Surv\&Tuts, vol. 19, no. 2, pp. 855-873, Secondquarter 2017.

[12] J. Guo, S. Durrani, X. Zhou, and H. Yanikomeroglu, "Massive machine type communication with data aggregation and resource scheduling," IEEE Trans. Commun., vol. 65, no. 9, pp. 4012-4026, Sep. 2017.

[13] A. Zemlianov and G. de Veciana, "Capacity of ad hoc wireless networks with infrastructure support," IEEE J. Sel. Areas Commun., vol. 23, no. 3 , pp. 657-667, Mar. 2005.

[14] C. Choi, F. Baccelli, and G. de Veciana, "Densification leveraging mobility: An IoT architecture based on mesh networking and vehicles," in Proc. ACM MobiHoc, 2018, pp. 71-80.

[15] S. Jain, R. C. Shah, W. Brunette, G. Borriello, and S. Roy, "Exploiting mobility for energy efficient data collection in wireless sensor networks," Mobile Networks and Applications, vol. 11, no. 3, pp. 327-339, 2006.

[16] G. Xing, T. Wang, W. Jia, and M. Li, "Rendezvous design algorithms for wireless sensor networks with a mobile base station," in Proc. ACM MobiHoc 2008, pp. 231-240.

[17] S. Forstall, G. N. Christie, R. E. Borchers, and K. Tiene, "Mobile device base station," June 2013, U.S. Patent 8,463,238.

[18] E. W. Saad, J. L. Vian, M. A. Vavrina, J. A. Nisbett, and D. C. Wunsch, "Vehicle base station," Dec 2014, U.S. Patent no. 8,899,903.

[19] M. Talluri, K. Agarwal, R. K. Mishra, and S. Garg, "Enhanced mobile base station," Mar 2018, U.S. Patent no. 9,913,095.

[20] C. Choi and F. Baccelli, "Spatial and temporal analysis of direct communications from static devices to mobile vehicles," IEEE Trans. Wireless Commun., vol. 18, no. 11, pp. 5128-5140, Nov. 2019.

[21] M. Grossglauser and D. N. Tse, "Mobility increases the capacity of ad hoc wireless networks," IEEE/ACM Trans. Netw., vol. 10, no. 4, pp. 477-486, Aug. 2002.

[22] W. Zhao, M. Ammar, and E. Zegura, "Controlling the mobility of multiple data transport ferries in a delay-tolerant network," in Proc. IEEE INFOCOM, vol. 2, 2005, pp. 1407-1418.

[23] A. Balasubramanian, B. Levine, and A. Venkataramani, "DTN routing as a resource allocation problem," in Proc. ACM SIGCOMM Computer Commun. Review, vol. 37, no. 4, 2007, pp. 373-384.

[24] K. Fall and S. Farrell, "DTN: an architectural retrospective," IEEE $J$. Sel. Areas Commun., vol. 26, no. 5, pp. 828-836, June 2008.

[25] M. Zorzi and R. R. Rao, "Geographic random forwarding (GeRaF) for ad hoc and sensor networks: energy and latency performance," IEEE Trans. Mobile Comput., vol. 2, no. 4, pp. 349-365, Oct. 2003. 
[26] A. Skordylis and N. Trigoni, "Delay-bounded routing in vehicular adhoc networks," in Proc. ACM MobiHoc, 2008, pp. 341-350.

[27] A. Abdrabou and W. Zhuang, "Probabilistic delay control and road side unit placement for vehicular ad hoc networks with disrupted connectivity," IEEE J. Sel. Areas Commun., vol. 29, no. 1, pp. 129139, Jan. 2011.

[28] C. Choi and F. Baccelli, "Modeling and optimization of direct communications from IoT devices to vehicles," in Proc. IEEE Globecom Workshops, Dec. 2018, pp. 1-7.

[29] - "Poisson Cox point processes for vehicular networks," IEEE Trans. Veh. Technol., vol. 67, no. 10, pp. 10 160-10 165, Oct 2018.

[30] S. Chandrasekharan, K. Gomez, A. Al-Hourani, S. Kandeepan, T. Rasheed, L. Goratti, L. Reynaud, D. Grace, I. Bucaille, T. Wirth et al., "Designing and implementing future aerial communication networks," IEEE Commun. Mag., vol. 54, no. 5, pp. 26-34, May 2016.

[31] X. Lin, V. Yajnanarayana, S. D. Muruganathan, S. Gao, H. Asplund, H.L. Maattanen, M. Bergstrom, S. Euler, and Y.-P. E. Wang, "The sky is not the limit: LTE for unmanned aerial vehicles," IEEE Commun. Mag., vol. 56, no. 4, pp. 204-210, Apr. 2018.

[32] S. D. Muruganathan, X. Lin, H.-L. Maattanen, Z. Zou, W. A. Hapsari, and S. Yasukawa, "An overview of 3GPP release-15 study on enhanced LTE support for connected drones," arXiv preprint arXiv:1805.00826, 2018.

[33] A. P. Cracknell, "UAVs: regulations and law enforcement," International J. Remote Sensing, vol. 38, no. 8-10, pp. 3054-3067, 2017.

[34] M. A. Ma'sum, M. K. Arrofi, G. Jati, F. Arifin, M. N. Kurniawan, P. Mursanto, and W. Jatmiko, "Simulation of intelligent unmanned aerial vehicle (UAV) for military surveillance," in Proc. IEEE ICACSIS, Sep. 2013, pp. 161-166.

[35] H. Arasteh, V. Hosseinnezhad, V. Loia, A. Tommasetti, O. Troisi, M. Shafie-khah, and P. Siano, "IoT-based smart cities: A survey," in Proc. IEEE EEEIC, June 2016, pp. 1-6.

[36] B. Galkin, J. Kibilda, and L. A. DaSilva, "Coverage analysis for low-altitude UAV networks in urban environments," in Proc. IEEE Globecom, Dec 2017, pp. 1-6.

[37] M. Mozaffari, W. Saad, M. Bennis, and M. Debbah, "Wireless communication using unmanned aerial vehicles (UAVs): Optimal transport theory for hover time optimization," IEEE Trans. Wireless Commun., vol. 16, no. 12, pp. 8052-8066, Dec 2017.

[38] G. Hattab and D. Cabric, "Energy-efficient massive IoT shared spectrum access over uav-enabled cellular networks," arXiv preprint arXiv:1808.08006, 2018.

[39] Y. Zeng, Q. Wu, and R. Zhang, "Accessing from the sky: A tutorial on UAV communications for 5G and beyond," arXiv preprint arXiv:1903.05289, 2019.

[40] W. Khawaja, I. Guvenc, D. W. Matolak, U. Fiebig, and N. Schneckenberger, "A survey of air-to-ground propagation channel modeling for unmanned aerial vehicles," IEEE Commun. Surv\&Tuts, vol. 21, no. 3, pp. 2361-2391, thirdquarter 2019.

[41] X. Zhou, S. Durrani, J. Guo, and H. Yanikomeroglu, "Underlay drone cell for temporary events: Impact of drone height and aerial channel environments," IEEE Internet Things J., vol. 6, no. 2, pp. 1704-1718, Apr. 2019.

[42] V. V. Chetlur and H. S. Dhillon, "Downlink coverage analysis for a finite 3-D wireless network of unmanned aerial vehicles," IEEE Trans. Commun., vol. 65, no. 10, pp. 4543-4558, Oct. 2017.

[43] Y. Li and L. Cai, "UAV-assisted dynamic coverage in a heterogeneous cellular system," IEEE Network, vol. 31, no. 4, pp. 56-61, July 2017.

[44] M. Mozaffari, W. Saad, M. Bennis, and M. Debbah, "Unmanned aerial vehicle with underlaid device-to-device communications: Performance and tradeoffs," IEEE Trans. Wireless Commun., vol. 15, no. 6, pp. 39493963, June 2016.

[45] M. M. Azari, F. Rosas, A. Chiumento, A. Ligata, and S. Pollin, "Uplink performance analysis of a drone cell in a random field of ground interferers," in Proc. IEEE WCNC, April 2018, pp. 1-6.

[46] F. Baccelli, B. Błaszczyszyn et al., Stochastic Geometry and Wireless Networks: Volume I Theory. Now Publishers, Inc., 2010, vol. 3, no. 3-4.

[47] F. Baccelli and A. Biswas, "On scaling limits of power law shot-noise fields," Stochastic Models, vol. 31, no. 2, pp. 187-207, 2015.

[48] F. Baccelli and P. Brémaud, Elements of queueing theory: Palm Martingale calculus and stochastic recurrences. Springer Verlag, 2013, vol. 26.

[49] M. Haenggi, R. K. Ganti et al., Interference in large wireless networks. Now Publishers, Inc., 2009, vol. 3, no. 2.
[50] F. Baccelli, B. Błaszczyszyn et al., Stochastic geometry and wireless networks: Volume II Applications. Now Publishers, Inc., 2010, vol. 4, no. 1-2.

[51] S. Boyd and L. Vandenberghe, Convex optimization. Cambridge university press, 2004. 